Title: The Molecular and Microbial Microenvironments in Chronically Diseased Lungs

Authors: Alexey V. Melnik ${ }^{1}$, Yoshiki Vázquez-Baeza ${ }^{2^{*}}$, Alexander A. Aksenov ${ }^{*}$, Embriette Hyde $^{3}$, Andrew C McAvoy ${ }^{4}$, Mingxun Wang ${ }^{5}$, Ricardo R. da Silva ${ }^{1}$, Ivan Protsyuk ${ }^{6}$, Jason V. $\mathrm{Wu}^{1}$, Amina Bouslimani ${ }^{1}$, Yan Wei Lim ${ }^{7}$, Tal Luzzatto-Knaan ${ }^{1}$, William Comstock ${ }^{1}$, Robert A. Quinn $^{1}$, Richard Wong ${ }^{8}$, Greg Humphrey ${ }^{3}$, Gail Ackermann ${ }^{3}$, Timothy Spivey ${ }^{9}$, Sharon S. Brouha $^{10}$, Nuno Bandeira ${ }^{5}$, Grace Y. Lin ${ }^{8}$, Forest Rohwer ${ }^{7}$, Douglas J. Conrad ${ }^{10}$, Theodore Alexandrov ${ }^{1,6,10}$, Rob Knight ${ }^{3,5,11,12}$, Pieter C. Dorrestein ${ }^{1}$, Neha Garg 1,4,13,14, \#

\title{
Author Affiliations:
}

${ }^{1}$ Collaborative Mass Spectrometry Innovation Center, Skaggs School of Pharmacy and Pharmaceutical Sciences, University of California, San Diego, La Jolla, CA 92093, USA.

2 Jacobs School of Engineering, University of California, San Diego, La Jolla, CA 92093, USA.

${ }^{3}$ Department of Pediatrics, University of California, San Diego, La Jolla, CA 92093, USA. Currently, Freelance Science Writer and Research Consultant.

${ }^{4}$ School of Chemistry and Biochemistry, Georgia Institute of Technology, Atlanta, GA 30332, USA.

${ }^{5}$ Department of Computer Science \& Engineering, University of California, San Diego, La Jolla, CA 92093, USA.

${ }^{6}$ Structural and Computational Biology Unit, European Molecular Biology Laboratory, Heidelberg 69117, Germany.

${ }^{7}$ Biology Department, San Diego State University, San Diego, CA, USA.

${ }^{8}$ Department of Pathology, University of California, San Diego, La Jolla, CA 92093, USA.

${ }^{9}$ Department of Radiology, University of California, San Diego, La Jolla, CA 92093, USA.

${ }^{10}$ Department of Medicine, University of California at San Diego, La Jolla, CA 92093, USA.

${ }^{11}$ UC San Diego Center for Microbiome Innovation, University of California, San Diego, La Jolla, CA 92093, USA

${ }^{12}$ Department of Bioengineering, University of California at San Diego, La Jolla, CA 92093, USA.

${ }^{13}$ Emory-Children's Center for Cystic Fibrosis and Airways Disease Research, Atlanta, GA 30322, USA.

${ }^{14}$ Center for Microbial Dynamics and Infection, Georgia Institute of Technology, Atlanta, GA 30332, USA.

\# To whom correspondence should be addressed: ngarg42@gatech.edu

*Equal contribution authors

\section{Author contributions:}

NG, DJC, FR, PCD created the idea for the work.

NG, RW, AB, TLK, YW, RAQ, GYL, DJC collected lung tissue

AM, NG, TS, SSB created 3D models

AM, NG picked coordinates on lung models

AM, RS, YVB performed statistical analysis

AM, EH, NG, GH, JG, GA, RK, AA, YVB performed 16S rRNA sequencing and analysis

MW, IP, RS, TA modified the tool 'ili

NG, JW extracted lung tissue for acquisition of mass spectrometry data

NG acquired mass spectrometry data

NG, WC performed microbiology experiments

AM, ACM, NG, IP, PCD analyzed mass spectrometry data

AM, NG, AAA, PCD wrote the paper 


\section{Abstract}

To visualize the personalized distributions of pathogens, chemical environments

52 including microbial metabolites, pharmaceuticals, and their metabolic products within and

53 between human lungs afflicted with cystic fibrosis, we generated 3D microbiome and

54 metabolome maps of six explanted lungs from three cystic fibrosis patients. These 3D spatial

55 maps revealed that the chemical environments are variable between patients and within the

56 lungs of each patient. Although the patients' microbial ecosystems were defined by the

57 dominant pathogen, their chemical diversity was not. Additionally, the chemical diversity

58 between locales in lungs of the same individual sometimes exceeded inter-individual variation.

59 Thus, the chemistry and microbiome of the explanted lungs appear to be not only personalized

60 but also regiospecific. Previously undescribed analogs of microbial quinolones and antibiotic

61 metabolites were also detected. Furthermore, mapping the chemical and microbial distributions

62 allowed visualization of microbial community interactions, such as increased production of

63 quorum sensing quinolones in locations where Pseudomonas was in contact with

64 Staphylococcus and Granulicatella, consistent with in vitro observations of bacteria isolated

65 from these patients. Visualization of microbe-metabolite associations within a host organ in

66 early-stage CF disease in animal models will help elucidate a complex interplay between the

67 presence of a given microbial structure, antibiotics, metabolism of antibiotics, microbial virulence

68 factors, and host responses.

70 Importance

71 Microbial infections are now recognized to be polymicrobial and personalized in nature.

72 A comprehensive analysis and understanding of the factors underlying the polymicrobial and

73 personalized nature of infections remains limited, especially in the context of the host. By

74 visualizing microbiomes and metabolomes of diseased human lungs, we describe how different

75 the chemical environments are between hosts that are dominated by the same pathogen and 
76 how community interactions shape the chemical environment, or vice versa. We highlight that

77 three-dimensional organ mapping are hypothesis building tools that allow us to design

78 mechanistic studies aimed at addressing microbial responses to other microbes, the host, and

79 pharmaceutical drugs.

80 Introduction

An increasing rate of infection from multi-drug resistant opportunistic pathogens has

82 become a significant burden in recent years. Proliferation of these pathogens due to overuse of

83 antibiotics, including those of last resort (1-4), is a threat to human health and is already

84 associated with increased mortality $(5,6)$. One reason for indiscriminate use of broad-spectrum

85 antibiotics and combination therapy in complex polymicrobial infections is the lack of knowledge

86 with regards to how microorganisms interact with each other, the host, and their chemical

87 environment, leading to strategies that target bacterial pathogens broadly. Herein, specific

88 microbial pathways that are involved in detrimental microbe-microbe interactions (7), microbe-

89 host interactions (8) and microbe-drug interactions (9) can serve as new targets for targeted

90 drug discovery. Thus, knowledge of such interaction-mediating microbial pathways and their

91 prevalence will shape the future of drug discovery. In this regard, even though we have begun

92 to appreciate the presence of multiple subpopulations by imaging community structures (10-12)

93 and by genome sequencing (13-15), information about the specific microbial pathways in

94 mediating the above mentioned interactions, molecular distribution of xenobiotic compounds,

95 and how such distributions are associated with specific microbial structures within the context of

96 a host is largely lacking.

97 We developed a methodology to map microbial and metabolite distributions in a human

98 lung in three dimensions to identify pathways that may be mediating microbial interactions and

99 to visualize the distribution of antibiotics in relation to microbial community structure (16). These

100 three dimensional organ maps allow visualization of chemical and microbial microenvironments

101 and consequently, may provide better insights into complex processes that take place within a 
102 host. Here we apply this methodology to elucidate spatial variation within and between the lungs

103 of three individuals afflicted with cystic fibrosis (CF).

104 CF is genetic disease caused by a mutation in the cystic fibrosis transmembrane

105 conductance regulator (CFTR) gene that results in defects of the encoded CFTR protein. The

106 primary function of CFTR protein is an ion channel that regulates liquid volume (mucus) on the

107 epithelial cells through secretion of chloride ions and inhibition of sodium absorption. Sticky

108 mucus accumulates in the upper airways and lungs of CF patients, and serves as growth

109 medium for various microbes, including opportunistic pathogens, resulting in chronic and

110 recurrent polymicrobial infections. In the 1930s, the life expectancy for someone diagnosed with

111 CF was only several years (17). Due to advances in modern medicine, including the use of

112 antibiotics and better clinical management of the disease, individuals with CF can now expect to

113 live on an average into their forties even though most patients are waitlisted for organ transplant

114 by the time they reach adulthood (18). Improved clinical management is partly made possible by

115 better understanding of the polymicrobial nature of the infections of the lung and development of

116 antibiotic-based clearance of chronic infections targeting the polymicrobial community (19).

117 However, the virulence of pathogens in microbial lung diseases such as CF, pneumonia,

118 tuberculosis, and chronic obstructive pulmonary disease is mostly studied in cultures derived

119 from pulmonary secretions and by genome sequencing, which does not represent complex in

120 vivo conditions. The failure in treating an infection in a complex organ such as human lung may

121 simply stem from the inability to treat a localized infection foci which can then spread to the

122 entire organ or become systemic as, for example, in case of infections caused by Burkholderia

$123(13,14,20,21)$. Understanding how the production of microbial small molecules involved in

124 pathogenicity and community interactions varies with lung biogeography leading to infection hot-

125 spots will enable the development of targeted antimicrobials and improved drug delivery

126 vehicles $(14,20,22)$. Thus, CF presents an important test case for improving disease 
127 management strategies for polymicrobial infections, given better understanding of community

128 structures and chemical environments within the host.

129 In this study, with patient consent, we mapped the chemical and microbial makeup of six

130 explanted lungs, removed during surgery from three CF patients, by using 3D volume

131 cartography to understand how microbes, microbial molecules, and medications are distributed

132 and metabolized throughout the entire organ, providing insights into microbe-microbe

133 interactions.

\section{Results and discussion}

The explanted lungs of three patients afflicted with CF were sectioned to inventory and

137 map the associated microbiome and metabolome in three dimensions onto lung models built

138 from CT-scans acquired prior to surgery (Methods) (16). To perform 16S rRNA gene analysis,

139 the tissue sections were swabbed, enabling detailed inventory of bacterial DNA present within

140 the patients' lungs (Table 1). We refer to our analysis of 16S rRNA gene as inventory of the

141 bacterial DNA and not the bacteria themselves, since lungs associated with CF are known to

142 contain a significant amount of DNA from dead cells as well as extracellular DNA (23). In total,

143 six lungs from three patients contained bacteria that spanned 40 genera (Supplementary Table

144 1). Bar plots of the most frequently amplified genera and their relative abundances pooled for all

145 anatomical locations are illustrated for each patient in Supplementary Figure 1. The relative

146 abundances of these genera in individual sections of each patient is available through 3D maps

147 (see below). The DNA of the most commonly occurring pathogenic organism in CF,

148 Pseudomonas aeruginosa, was detected at highest frequency throughout the lungs of patients 1

149 and 3, whereas patient 2 was dominated by DNA from the emerging pathogen

150 Stenotrophomonas. Even though the microbial population within CF-associated lungs can be

151 heterogeneous (24), dominance of a single pathogen at end-stage CF disease has been

152 described extensively in previous studies (25-27). 
The PCoA analysis of microbiome data with weighted UniFrac distance showed

154 clustering between both lungs of patient 1 and the right lung of patient 3 along the first two

155 principal axes (Supplementary Figure 2a). Samples from the left lung of patient 3 clustered

156 separately and the comparison of 10-most abundant OTUs further highlighted the differences

157 between the microbial communities present in the left lung of patient 3 (Supplementary Figure

$1582 \mathrm{~b}$ and Supplementary Figure 3). Apart from the dominant pathogen, the overall microbiome

159 between and within patients was different along the second and third axis (Supplementary

160 Figure 2 c,d). The Canberra distance metric yielded less apparent but visible separation of

161 patients' microbiome data in PCoA space (Figure $1 \mathrm{a}, \mathrm{b}$ ); in some cases this variation within

162 patient's own lungs was found to be greater than between patients (Figure 1b). A more

163 homogeneous clustering patterns is observed in the $16 \mathrm{~S}$ data when the unweighted UniFrac

164 metric, a phylogenetically informed metric, was applied (Supplementary Figure 4). The

165 differences arise due to the qualitative nature of unweighted UniFrac and the quantitative

166 comparisons possible with the Canberra metric. 
a)

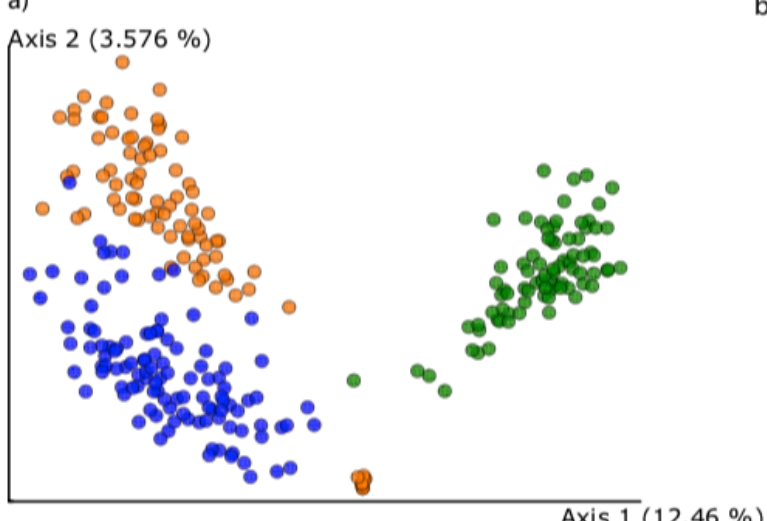

c)

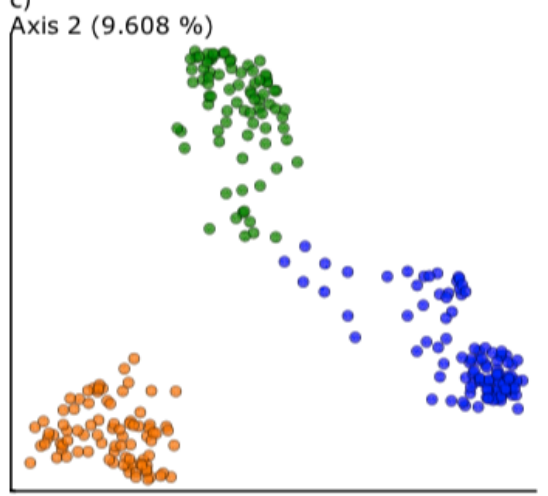

Axis $1(12.51 \%)$ b)

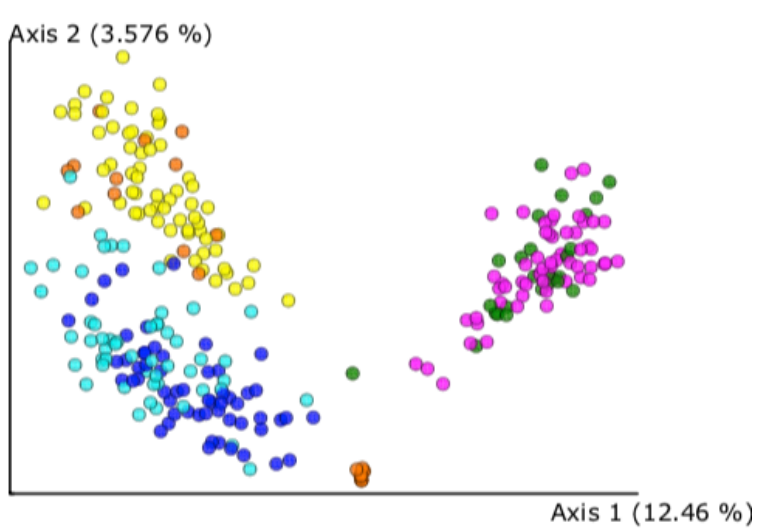

d)

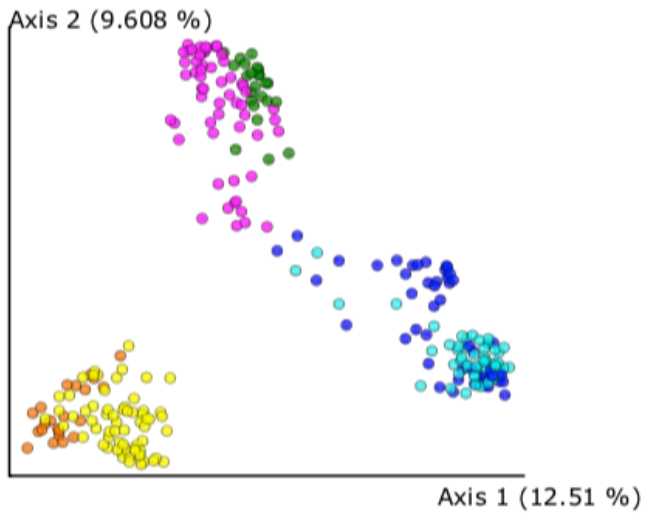

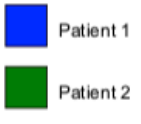

Patient 3 data, respectively.

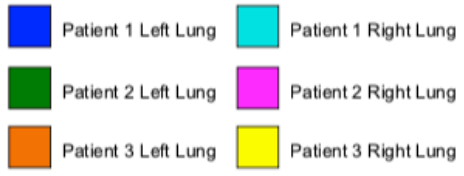

Figure 1. Principal coordinate plot of metabolome and microbiome from lungs of three patients in the study. Visualization was performed in the Emperor software using Canberra distance metric (28). a), b) PCoA plots of $16 \mathrm{~S}$ rRNA sequencing and c), d) mass spectrometry

To map the relative frequency of microbes onto the $3 \mathrm{D}$ lung models, we used our previously described methodology (16). The distribution of prevalent (Pseudomonas and Staphylococcus) and emerging (Stenotrophomonas and Achromobacter) microbes in the CFassociated lungs is displayed in Figure 2. Although nearly uniform distribution of dominant

177 pathogens (Pseudomonas in patients 1 and 3, Stenotrophomonas in patient 2) was observed, 178 all other microbes were distributed unevenly, often relegated to niche spots. For example, 179 Achromobacter was mainly localized in the apex of the right lung of patient 3 while 
180 Staphylococcus was present in the lower lobe of both lungs of patient 1 , at the apex of the lungs

181 of patient 2 and in the middle and lower lobes of the lungs of patient 3 . The dominant pathogen,

182 Stenotrophomonas, showed uniform distribution in the lungs of the patient 2 and differential

183 distributions in the lungs of patients 1 and 3 (Figure 2). A degree of stratification is expected

184 based on the availability of oxygen: Achromobacter and Stenotrophomonas are strict aerobes

185 whereas Staphylococcus and Pseudomonas are facultative anaerobes residing as biofilms in

186 airway mucus of CF patients with the potential of undergoing anaerobic metabolism (29).

187 Furthermore, patients 1 and 3 not only share the dominant pathogen, their microbial

188 communities are also more similar to each other than either is to that of the patient 2

189 (Supplementary Figure 3 and 5). Selection pressures from competing microbes and chemical

190 microenvironments including antibiotic distributions, further leads to stratification of niches

191 occupied by specific organisms. To compare the microbial and chemical environments, we next

192 annotated the mass spectrometry data acquired on the tissue sections and mapped it onto the

193 3D models of the lungs of these patients (see below). 

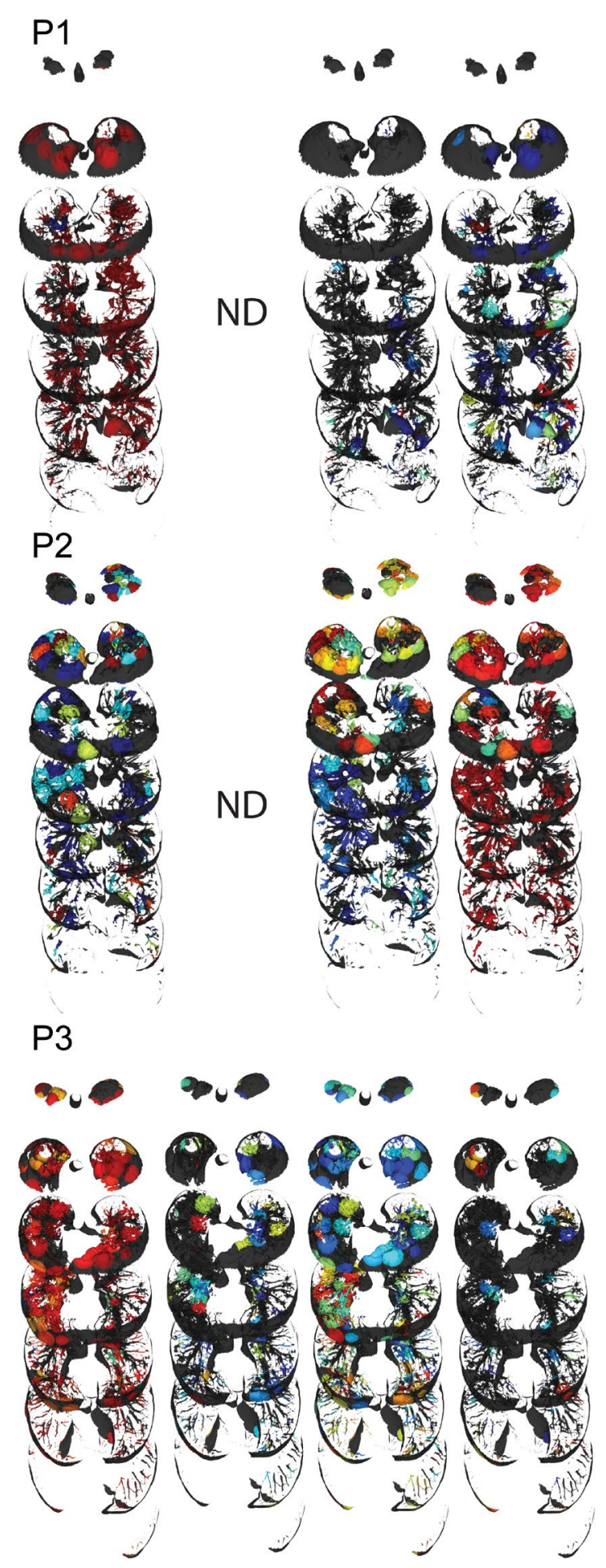

Figure 2. Distribution of microorganisms. Distribution of Pseudomonas, Achromobacter, Staphylococcus, and Stenotrophomonas (right to left) are shown for all three patients. P1 patient one, P2 - patient two and P3 - patient three, ND - not detected. Intensity scale is provided at the bottom right. Full visualizations of microbial maps can be accessed via links: patient 1 , patient 2 and patient 3 . 
To annotate molecular ions detected using high resolution mass spectrometry (MS)based untargeted approach, molecular network analysis was performed using the Global

202 Natural Product Social Molecular Networking (GNPS) infrastructure (30). Molecular networking

203 allows for reduction and organization of the overwhelming amount of chemical information

204 generated (in terms of mass spectra) in a high-resolution untargeted MS approach. The data

205 reduction is performed by combining and displaying the identical MS/MS spectra as a single

206 node and by displaying similar spectra as connected nodes $(30,31)$. Similarities in MS/MS

207 spectra relate to similarities in chemical structures, so oftentimes such connected nodes

208 represent chemical and biological transformations of a molecule. In this study, 676,451 MS/MS

209 spectra were filtered and merged into consensus spectra, producing 9,874 nodes (Figure 3a).

210 The patient-specific molecules were displayed by assigning a specific color to each patient in

211 the molecular network analysis (Figure 3a). In addition to annotating known compounds,

212 molecular networking revealed related molecules that differ by oxidation, methylation,

213 acetylation, hydroxylation, glycosylation, chain length, and saturation of alkyl chains, which

214 enabled identification of previously undescribed metabolites of administered pharmaceuticals

215 and microbial quinolones, as described below for azithromycin and Pseudomonas aeruginosa

216 quinolones. The frequency of detection of the antibiotics across patients' samples is shown in

217 Figure 3b, with corresponding clusters from the full network displayed for each antibiotic. The

218 nodes in antibiotic cluster represent the metabolic transformations of the antibiotic. Thus,

219 molecular networking provides a glimpse into metabolic processes. The resulting molecular

220 network revealed that among three patients, remarkably, only about $27.6 \%$ of detected

221 molecular features were shared, highlighting the diversity of chemistry present in diseased

222 human lungs (Figure 3c). All three patients in this study had different mutations in the CFTR

223 gene (see Materials and Methods) and patients 2 and 3 were also diagnosed with CF-related

224 diabetes. Two of the three patients suffered from chronic infections by Pseudomonas

225 aeruginosa. Thus, various factors may play a role leading to the observed chemical diversity, 
226 which may arise from microbial (e.g., virulence and quorum sensing metabolites such as

227 quinolones), host (e.g., bile acids, amino acids, sugars, eukaryotic lipids, fatty acids, sterols,

228 peptides, immune-related molecules), and xenobiotic molecules. The diversity of these

229 metabolites in CF sputum has been previously characterized and many of the same compounds

230 were also found in the lung tissue in this study (32). 

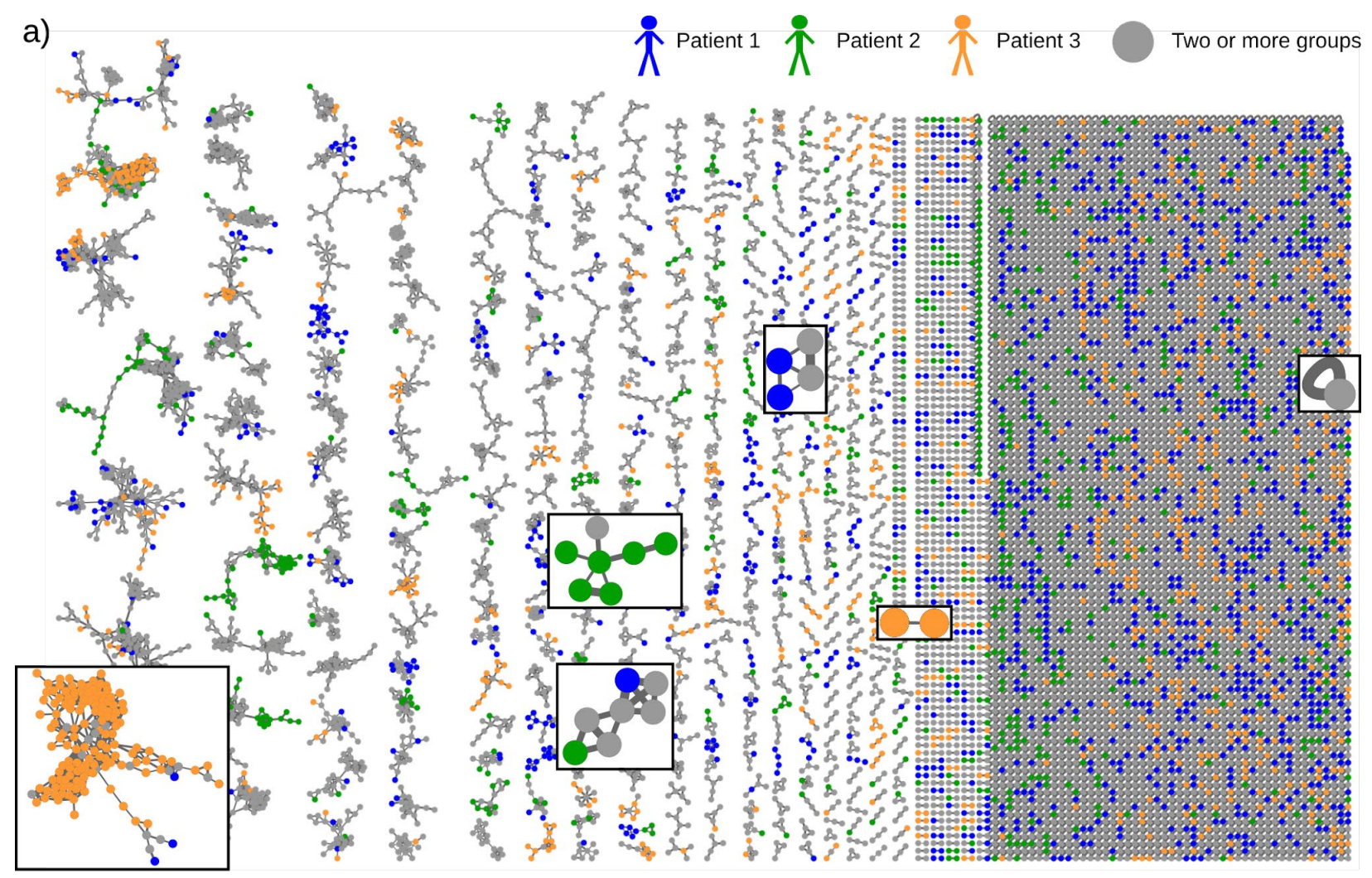

b)
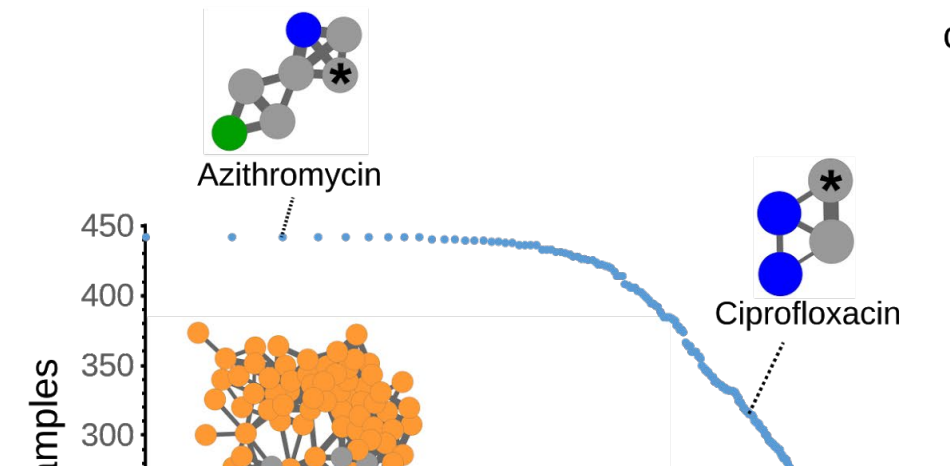

c)

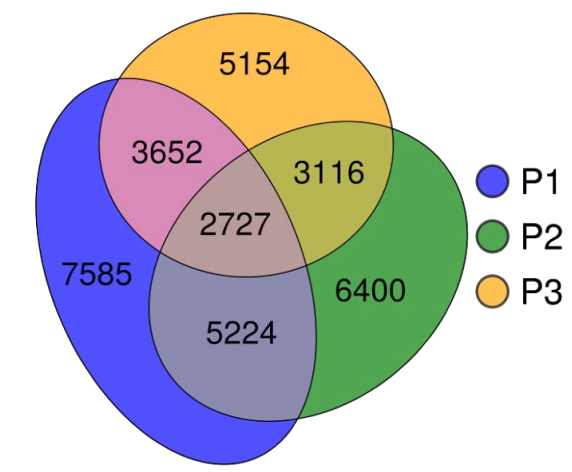

a) The molecular network is color coded by patients (patient 1 (blue), patient 2 (green), and patient 3 (orange). The network clusters corresponding to antibiotics are highlighted in boxes. b) The number of samples that contain a given consensus MS/MS spectra (represented as 
node in Figure 3a) are plotted. The frequency of occurrence of antibiotics detected in this dataset is highlighted on the plot. The number of nodes in a cluster is reflective of detected transformations of the parent compound. The node of parent compound is highlighted by asterisks. The fragmentation patterns of the most frequently observed drug - azithromycin and its analogs are described in Supplementary Figure 7; the large number of nodes in Piperacillin cluster stems from its structural similarity to small peptidic compounds abundant in biological samples and its inherent chemical reactivity with biological molecules (33). c) Venn diagram of the overlap of consensus fragmentation spectra between three patients is shown.

A Procrustes analysis of metabolomics data and 16S rRNA data with closed-reference OTU picking revealed a close association between the microbiome and metabolome in the lung samples (Mantel test $r$ statistic $=0.45, P<0.001, n=277)($ Supplementary Figure $6(a, b)$ ). This analysis suggests that the microbial composition of each sample in large part is associated with the corresponding chemical diversity. Additionally, Procrustes analysis performed on metabolomics and 16S rRNA with deblurred sOTUs (34) resulted in the same trend (Supplementary Figure $6(\mathrm{c}, \mathrm{d})$, Mantel test $r$ statistic $=0.38, P<0.001, n=263$ ). A PCoA plot of the metabolome data with Canberra distance showed that a vast chemical diversity exists not only between the patients (Figure 1c), but also within a patient's own lungs (Supplementary table 2). This suggests that the chemical makeup of the patients with CF disease is highly personalized and that a single CF lung contains unique chemical microenvironments that provide different niches for microbial pathogens to live in. While metabolic diversity between patients in relation to disease state is previously described $(32,35)$, mechanisms leading to such diversity within the lungs remain poorly understood.

One of the additional benefits of an untargeted metabolomics analysis approach is the ability to track the medications that are taken by the patient, as medical records can oftentimes be incomplete and/or inaccurate due to lack of patient compliance, as well as to identify metabolic transformations of the medications. For example, in the present study, in addition to the prescribed medications from the clinical records (different antibiotics, bronchodilators, two medications for digestive health, medications given during surgery and over-the-counter medications that are used as cough suppressants), antihistamines and multiple over the counter 
267 medications have been detected (Table 1). Detailed knowledge of extant exogenous 268 compounds in tissues of interest is important, among other things, to evaluate their effect on the 269 microbiome and microbial interactions for better understanding disease etiology. Another 270 advantage of a molecular networking approach for untargeted metabolomics data analysis is

271 that it allows for postulating structures for unknown compounds, nodes of which are connected

272 to nodes of known compounds (annotation propagation), and is therefore very useful for

273 identifying drug metabolites (Figure 3b). The distributions of drugs and the metabolites can then

274 be evaluated by $3 D$ cartography even in the absence of a stable isotope tracer. Using a

275 molecular networking approach in this study, unknown metabolites that have never been

276 reported before in blood or tissue of humans and animals were detected (Supplementary Figure

277 7). The unknown metabolite of Azithromycin $(\mathrm{m} / \mathrm{z} 382.26)$ is annotated as methylated-

278 azithromycin where the methylation, based on the analysis of the fragmentation data, occurs in

279 the core macrolide ring structure of azithromycin and another unknown metabolite is proposed

280 to have oxidation in the macrolide ring (Supplementary Figure 7). These modifications of core

281 macrolide structure of azithromycin have not been described previously and their biological

282 activities are unknown. Although these metabolites were not detected in in vitro cultures of

283 microbes isolated from these patients in the presence of azithromycin, the possibility that these

284 are microbially-derived warrants further investigation and cannot be ruled out. Specific in vivo 285 conditions may be necessary for regulation of microbial genes involved in antimicrobial 286 metabolism. 
Table 1. The medications detected in the MS data and time of administration prior to the day of lung explantation surgery. C refers to: "continuously administered"

\begin{tabular}{|c|c|c|c|c|c|c|}
\hline \multirow{2}{*}{ Medications } & \multicolumn{3}{|c|}{ Patient } & \multirow{2}{*}{\multicolumn{3}{|c|}{$\begin{array}{c}\text { Time before } \\
\text { surgery (days) }\end{array}$}} \\
\hline & 1 & 2 & 3 & & & \\
\hline \multicolumn{7}{|l|}{ Antibiotics } \\
\hline 1. Trimethoprim & & $\bullet$ & $\circ$ & - & 8 & - \\
\hline 2. Sulfamethoxazole & & $\bullet$ & & - & 8 & - \\
\hline Acetylsulfamethoxazole & & 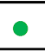 & & & & \\
\hline Sulamethoxazole-glucuronide & & $\bullet$ & & & & \\
\hline Sulfamethoxazole metabolite m/z 502.26 & & $\bullet$ & & & & \\
\hline 3. Azithromycin & - & - & - & $\mathrm{C}^{*}$ & C & 10 \\
\hline Descladinose-azithromycin & $\bullet$ & $\bullet$ & 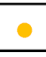 & & & \\
\hline 3"-O-desmethyl-azithromycin & $\bullet$ & $\bullet$ & 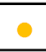 & & & \\
\hline 3'-N-desmethyldescladinose-azithromycin & & $\bullet$ & $\circ$ & & & \\
\hline Methyl-azithromycin unknown & $\bullet$ & $\bullet$ & $\circ$ & & & \\
\hline Descladinose-azithromycin (oxidation -2Da, unknown) & - & $\bullet$ & 0 & & & \\
\hline 4. Piperacillin & - & & 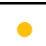 & 6 & - & 1 \\
\hline Desethylpiperacillin & - & & $\circ$ & & & \\
\hline 5. Ciprofloxacin & $\bullet$ & & $\circ$ & 6 & - & 1 \\
\hline 6. Ceftazidime & & & $\circ$ & & - & 1 \\
\hline 7. Minocycline & $\bullet$ & & & 2 & - & - \\
\hline 8. Linezolid & & $\bullet$ & & - & $\mathrm{X}$ & - \\
\hline \multicolumn{7}{|l|}{ Bronchodilator } \\
\hline 1. Pseudoephedrine & & $\bullet$ & & - & - & - \\
\hline 2. Albuterol (bronchodilator) & $\bullet$ & & & 6 & & \\
\hline \multicolumn{7}{|l|}{ Digestive Health } \\
\hline 1. Famotidine & - & & & 6 & - & - \\
\hline 2. Metoclopramide & & & $\cdot$ & - & - & 1 \\
\hline \multicolumn{7}{|c|}{ Surgery (sedation, anxiolytic, paralytic) } \\
\hline 1. Fentanyl (for surgical pain) & 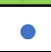 & & $\circ$ & & & \\
\hline 2. Haloperidol (ICU) & $\bullet$ & & & & & \\
\hline 3. Dihydrohaloperidol & $\bullet$ & & & & & \\
\hline 4. Mycophenolate (immune suppressant) & & 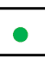 & & & & \\
\hline 5. Midazolam & & 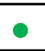 & $\circ$ & & & \\
\hline Hydroxymidazolam & & $\bullet$ & & & & \\
\hline Hydroxymidazolam glucuronide & & $\bullet$ & & & & \\
\hline 6. Rocuronium bromide & $\bullet$ & $\bullet$ & $\circ$ & & & \\
\hline 7. Vecuronium bromide & & & $\circ$ & & & \\
\hline 8. Ondansetron & & & 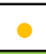 & & & \\
\hline \multicolumn{7}{|l|}{ Others } \\
\hline 1. Diphenhydramine & & $\bullet$ & 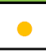 & - & 8 & 10 \\
\hline 2. Amitryptiline (antidepressant) & - & & & $\mathrm{X}$ & - & - \\
\hline 3. Loratadine & $\bullet$ & & & 6 & - & - \\
\hline Desloratadine (active ingredient of loratadine, antihistamine) & $\bullet$ & & & & & \\
\hline 4. Chlorhexidine (disinfectant) & 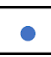 & $\bullet$ & $\circ$ & - & - & - \\
\hline
\end{tabular}


As with the microbial heterogeneity, we have observed differences in metabolome

293 distributions. Molecular networking and 3D volume cartography of the antibiotics revealed

294 patient-specific metabolism and drug distributions (Figure 4 and Supplementary Figure 8). The

295 distribution of antibiotics was also found to be different between the left and right lungs of the

296 same patient. For example, the antibiotic Piperacillin and its metabolites were abundant in the

297 upper lobes of the right lung of patient 3 but present in relatively lower abundance in the right

298 lung of this patient (Figure 4). In patient 3, there was higher penetration of piperacillin in the

299 upper and middle lobes and poor penetration in the lower lobes of both lungs. Similarly, the

300 antibiotic Linezolid detected in patient 2 had lower relative abundance in the lower lobe of the

301 right lung (Supplementary Figure 8). Overall, the drug metabolites largely follow the same

302 distribution as parent drug except for the glucuronidated metabolite of sulfamethoxazole

303 (Supplementary Figure 8), indicating that metabolism may not be a significant contributing factor

304 for observed uneven distribution of detected antibiotics. Differential vascularization and tissue

305 necrosis also contributes to non-uniform drug penetration in severe end-stage CF disease. 
bioRxiv preprint doi: https://doi.org/101101/676148; this version posted June 19,2019 . The copyright holder for this preprint (which was not

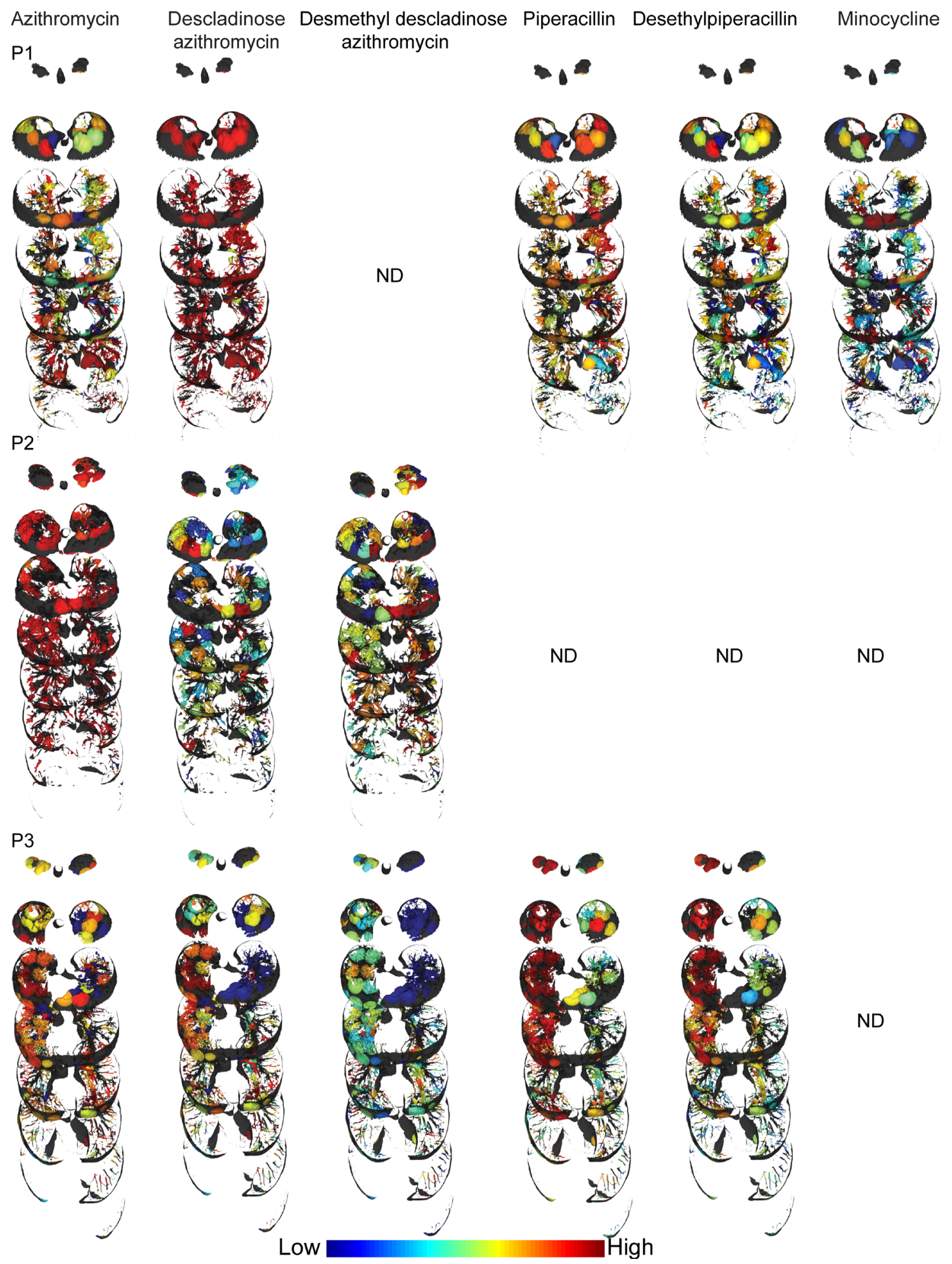

Figure 4. Distribution of selected antibiotics and the metabolites. P1 - patient 1, P2 patient 2 and P3 - patient 3, ND - not detected. Intensity scale is provided at the bottom (distribution of additional antibiotics and their metabolites is provided in Supplementary Figure 8). Full visualizations of metabolite maps can be accessed via links: patient 1, patient 2 and patient 3. 
To directly link microbiome and metabolome information and identify associations of compounds detected in the lung tissue with specific microbes, isolates from lungs of all patients were obtained from the clinical lab, cultured directly from swabs of the lung tissue, and MS data was acquired on the organic extracts of the in vitro cultures using the same protocol as employed for the tissue extracts. Molecular networking of MS/MS data from culture extracts and

317 tissue extracts provided insights into which molecules are shared between microbes and the

318 human host (Supplementary Figure 9). These molecules include microbe-specific virulence

319 factors, as well as various other molecules such as lipids, fatty acids, amino acid metabolites,

320 dipeptides and tripeptides. Similar to our previously reported observation for one CF lung of a

321 single CF patient $(16,32)$, a larger diversity of quinolones was detected in cultured isolates as

322 opposed to a smaller diversity of quinolones detected in the lung tissue of all patients dominated

323 by Pseudomonas in this study, including a quinolone at $\mathrm{m} / \mathrm{z} 268.170$ that has never been

324 reported before (Figure 5). Based on $\mathrm{MS}^{1}$ and $\mathrm{MS}^{2}$ data, the structure of this quinolone is

325 proposed to contain two double bonds in the alkyl side chain as opposed to single double bond

326 found in unsaturated quinolones described in the literature (36) (Supplementary Figure 10). To

327 gain further insight into the variation in the distribution of quinolones in the patients dominated

328 with Pseudomonas, we investigated the distribution of quinolones directly within the lungs of

329 these patients (Figure $5 b$ and Supplementary Figure 11). Previously, we reported that the

330 quinolones were prevalent at the upper lobe of the left lung of a single patient (16). In this study

331 quinolones were found to be exclusively present at the upper lobe of lungs of patient 1 and only

332 in the middle of the lungs of patient 3. This indicates that patients dominated by Pseudomonas

333 show individualized phenotypes with respect to the expression of these quorum sensing

334 molecules. Furthermore, rhamnolipids, the Pseudomonas' biosurfactant, were not detected in

335 the lungs of patient 1 and 3 in this study but were detected in our previous study (16). Patient-

336 specific production of rhamnolipids has been reported previously by culturing isolates in the

337 laboratory but not directly from infected tissue (13). Such compartmentalization of microbial 
338 activity within patients, as well as variation between patients is a hallmark of complexity that is

339 inherent to polymicrobial infection in a complex organ; in the present case, a CF lung. Direct

340 visualization of the individual phenotypes in diseased organs enables informed understanding of

341 divergent evolution as well as the spatial molecular environment within a host. 
a)

Isolates

Shared between isolates and Patients 1,3

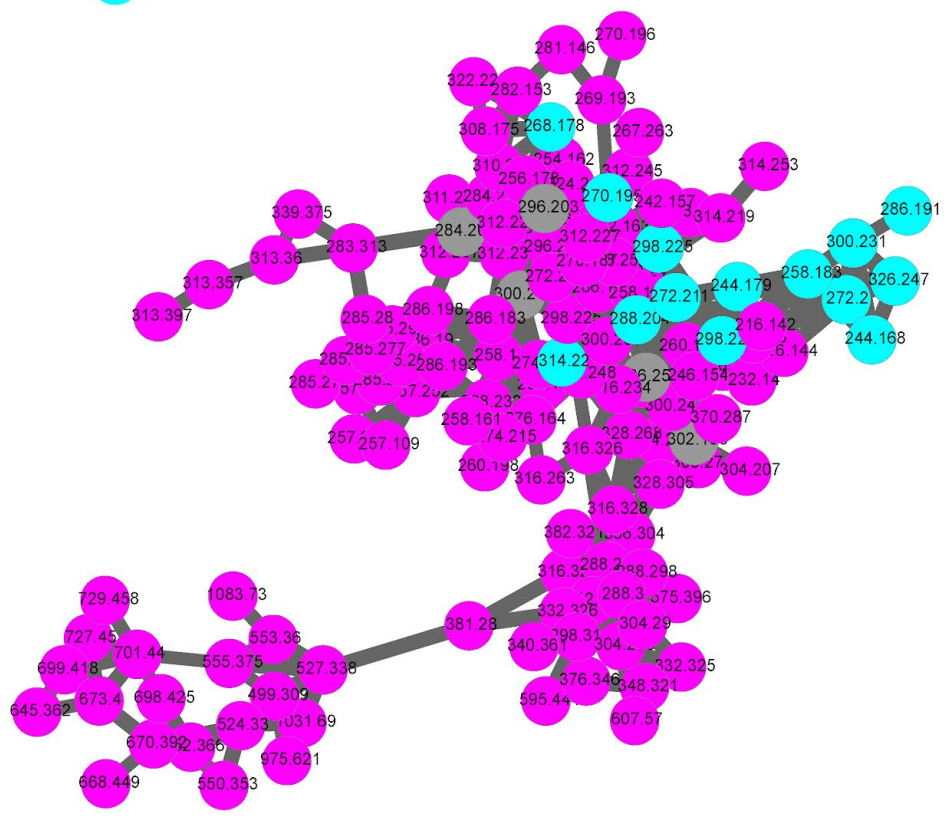

List of annotated quinolones:

HHQ C7 (m/z 244.179)

UHQ C11:1 (m/z 298.225)

NHQ C9 (m/z 272.210)

NHQ C9:2 putative (m/z 268.178)

NHQ C9:1 (m/z 270.195)

NQNO C9 (m/z 288.196)

UHQ C11 (m/z 300.232)

NQNO C9:1 (m/z 286.191) b)

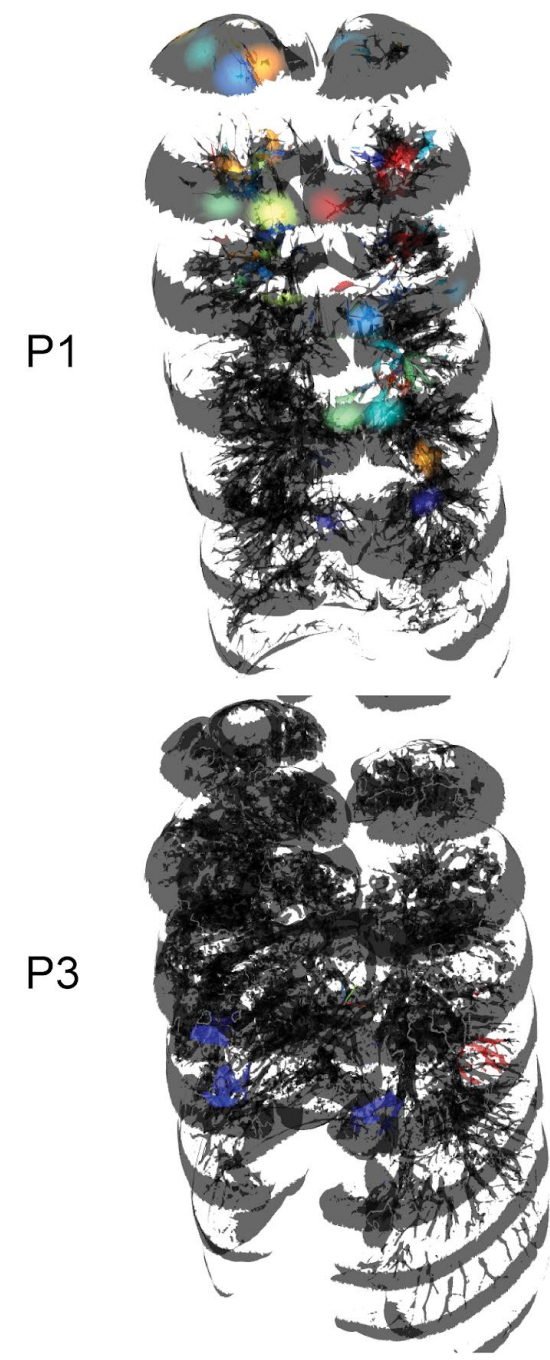

c)

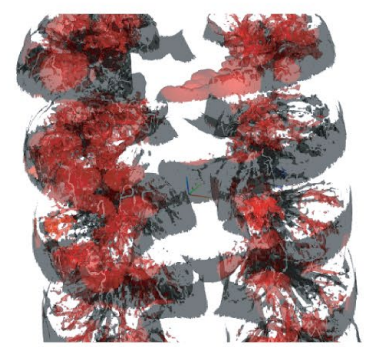

Pseudomonas

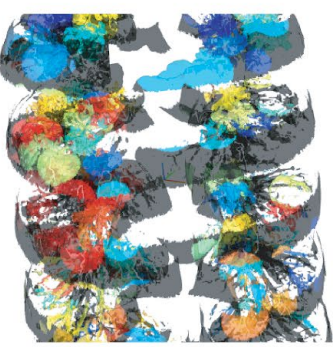

Staphylococcus

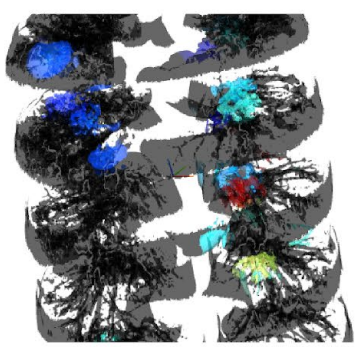

Granulicatella

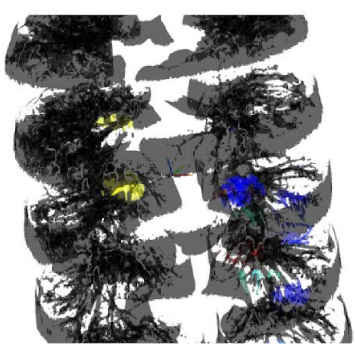

$\mathrm{NHQ}$
342

Figure 5. Molecules produced by $\boldsymbol{P}$. aeruginosa in patients 1 and 3 . a) The molecular network cluster of quinolones detected in the lung tissue of patient 1 and 3 and in vitro microbial cultures of Pseudomonas isolated from sputum and the swabs collected from lung sections is shown. b) The distribution of the quinolone $\mathrm{HHQ}$ is shown in patients 1 and 3 . All the other quinolones showed similar distributions in these patients (Supplementary Figure 11). c) Inset of the distribution of Pseudomonas, Staphylococcus, Granulicatella and the Pseudomonas quinolone $\mathrm{NHQ}$ in patient 3 suggestive of upregulation in quinolone production by 
Pseudomonas in the regions where interactions of Pseudomonas with Staphylococcus, Granulicatella and possibly other microbes take place. In agreement with this observation, the production of $\mathrm{HHQ}$ and $\mathrm{NHQ}$ was also found to increase in co-cultures of Pseudomonas and Staphylococcus compared to Pseudomonas grown alone under identical conditions (Supplementary Figure 12).

The spatial co-distribution of microorganisms, antibiotics and microbial molecules were investigated to establish microbe-metabolite interactions. Although the presence of a single dominant pathogen renders correlation analysis rather uninformative, several trends have been observed. In particular, the distribution of certain microorganisms such as Staphylococcus and Granulicatella were found to be associated with the distribution of quinolones produced by

362 Pseudomonas in patient 3, as shown for NHQ in Figure 5c. We have recently shown that Staphylococcus aureus isolated from a CF patient increases quinolone and biofilm production by co-isolated Pseudomonas in vitro (37). Similarly, mixing cultures of the Pseudomonas and Staphylococcus isolated from patient 3 in this study showed increased production of $\mathrm{HHQ}$ and NHQ when compared to Pseudomonas grown alone under identical conditions (Supplementary

367 Figure 12a). This observation indicates that the production of quinolone molecules is in part also modulated by microbial interactions present in a polymicrobial infection. The complexity of these microbial interactions is further increased as antibiotics cause perturbations of microbial communities reflected by suppression of the virulence factors. Variation in production of

371 quinolones by patient isolates of Pseudomonas was observed upon exposure to sub-MIC 372 concentrations (Supplementary Figures 12b). This, and the other observations reported here 373 support the hypothesis that not only are genetic changes responsible for changing metabolism,

374 but microbial interactions in conjunction with multiple other factors including sub-MIC 375 concentrations of antibiotics and perhaps other xenobiotics may also play a role and call for the 376 design of specific studies investigating these phenomena in multiple patient isolates. Thus, it is

377 reasonable to hypothesize that both specific microbial interactions in the lungs and differential 378 abundances of antibiotics could result in metabolic divergence, creating isolated regions of 
379 enhanced biofilm formation and tissue damage that is often observed in CF patients by chest X-

380 rays and CT-scans. Application of advanced techniques such as Ultra-High-Resolution

381 Computed Tomography in conjunction with the approach presented here could be a focus of

382 future studies (38).

\section{Conclusion}

Cystic fibrosis is a devastating genetic disease which affects tens of thousands of people worldwide. In this work, we presented the findings of spatial distributions of microbes,

387 medications, and their metabolites throughout lungs of three patients afflicted with CF. We have

388 found that although the microbiome is predominantly patient-specific, the chemical differences between locations within patient's own lungs may be greater than inter-patient variations. Indepth analyses revealed differential drug penetration, metabolism of prescribed medications,

391 and microbial compartmentalization resulting in metabolic divergence governed by local

392 microbial interactions. Mapping of microbial communities and localized chemistries allowed for

393 visualization of interactions among community members, such as production of quinolones by 394 Pseudomonas when present in a community structure with other microbes such as 395 Staphylococcus or Granulicatella. Visualization of such local infection loci highlight the 396 importance of development of effective drug delivery approaches. Considering recent advances

397 in the development of small-scale robots (as small as few micrometers) that can non-invasively 398 access confined spaces (39), targeted access of internal tissues as well as precision delivery of 399 drug payloads may become feasible in the near future. In general, a paradigm shift of 400 considering localized regions of divergent microbial and chemical distributions is an important 401 next step for effective disease management of polymicrobial infections. 
bioRxiv preprint doi: https://doi.org/10.1101/676148; this version posted June 19, 2019. The copyright holder for this preprint (which was not certified by peer review) is the author/funder, who has granted bioRxiv a license to display the preprint in perpetuity. It is made available under aCC-BY-NC-ND 4.0 International license.

Supplementary Figures and Tables:

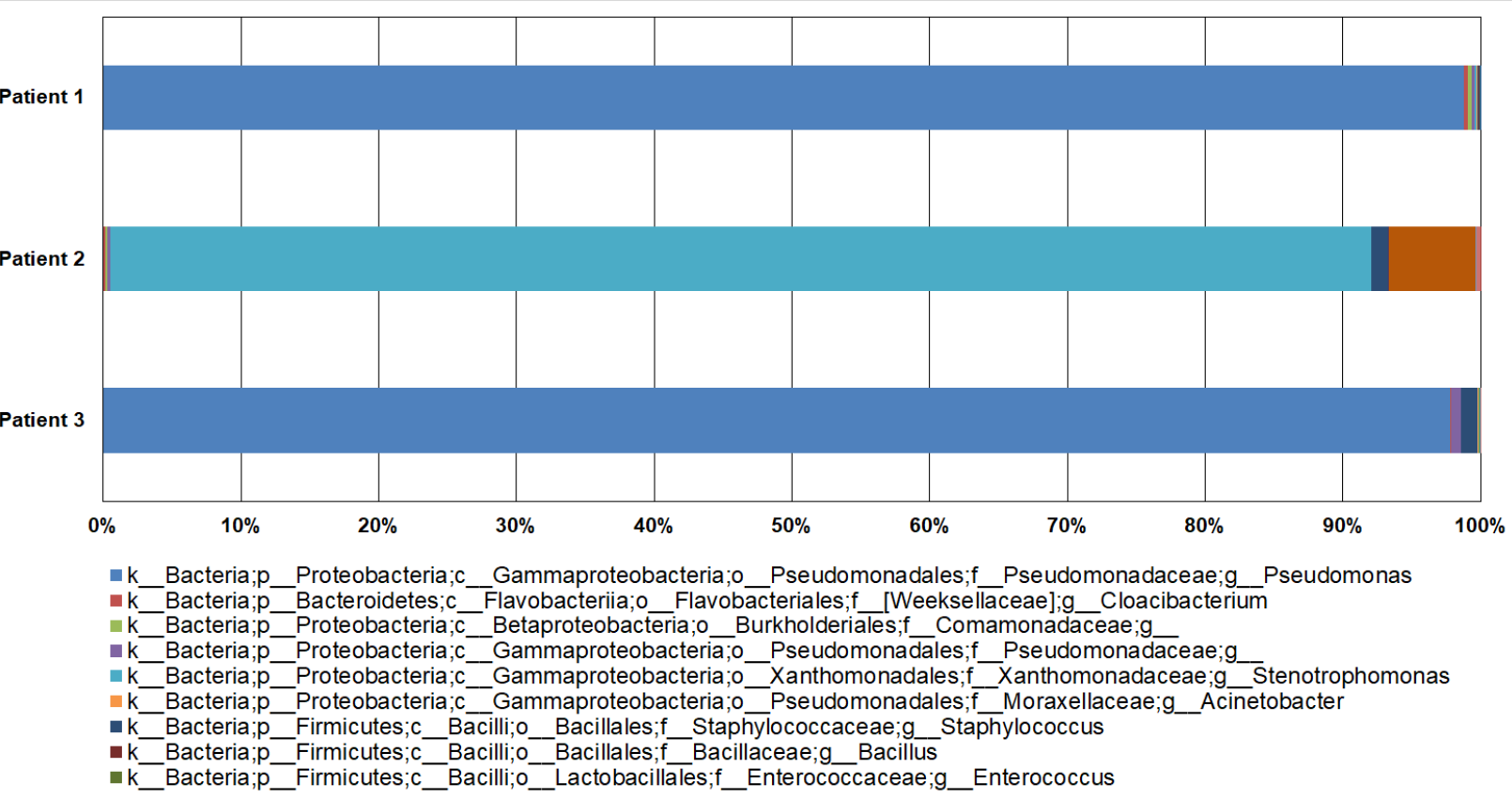

Supplementary Figure 1. The bar charts of taxonomic summary of all samples for each patient is shown. The legend for 9 most abundant taxa is shown. The complete taxonomic summary is available in Supplementary table 1. The complete description of all OTU's for each individual sections are available through spatial maps: patient 1, patient 2 and patient 3. 
a)

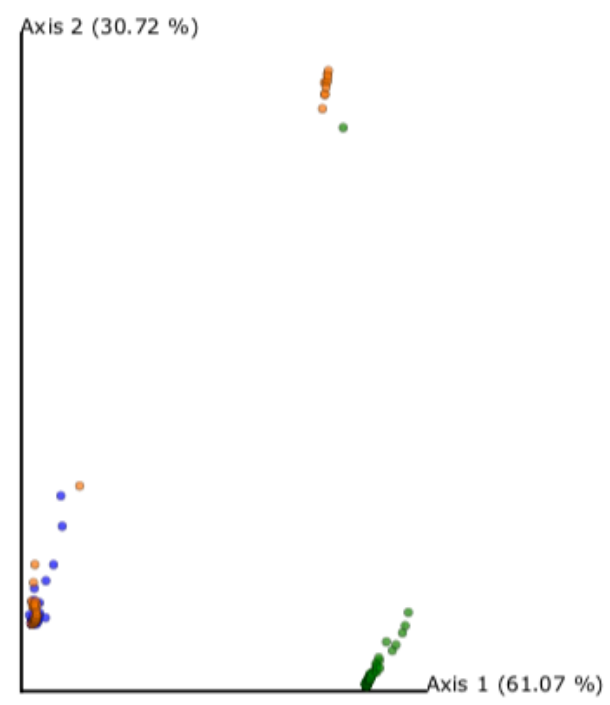

c)

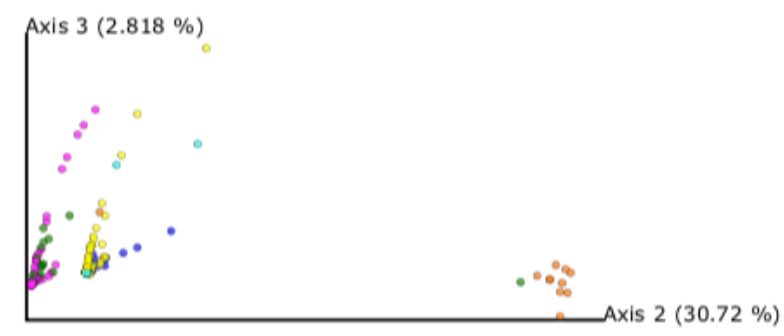

Patient 1

Patient 2

Patient 3

b)

d)
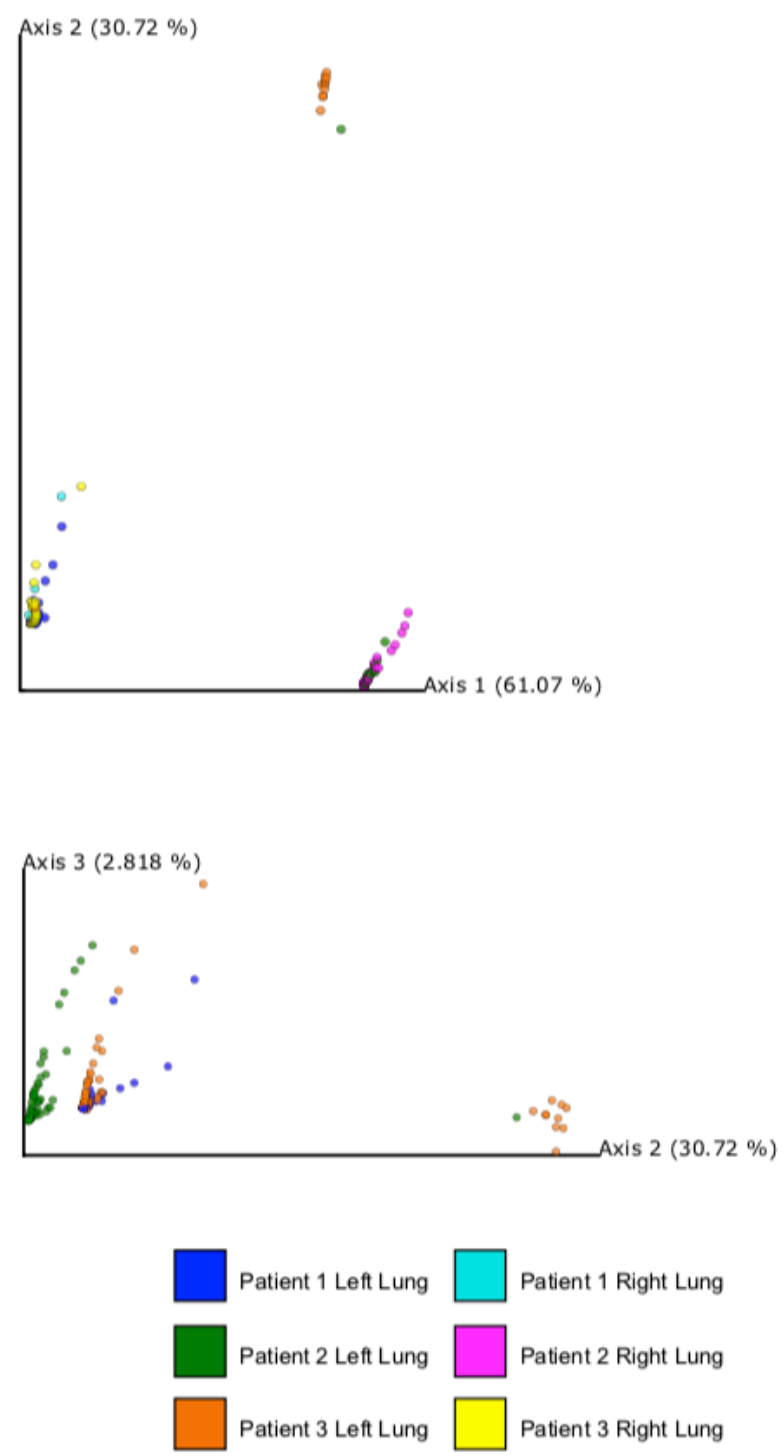

409
Supplementary Figure 2. PCoA plot on Weighted Unifrac distance of 16S rRNA data from three patients is shown (40). a) and b) first and second principal component is shown. c) and d) second and third principal component is shown. 
414

415

416

417

418

419

420

421

422

423

424

425

426

427

428

429

430

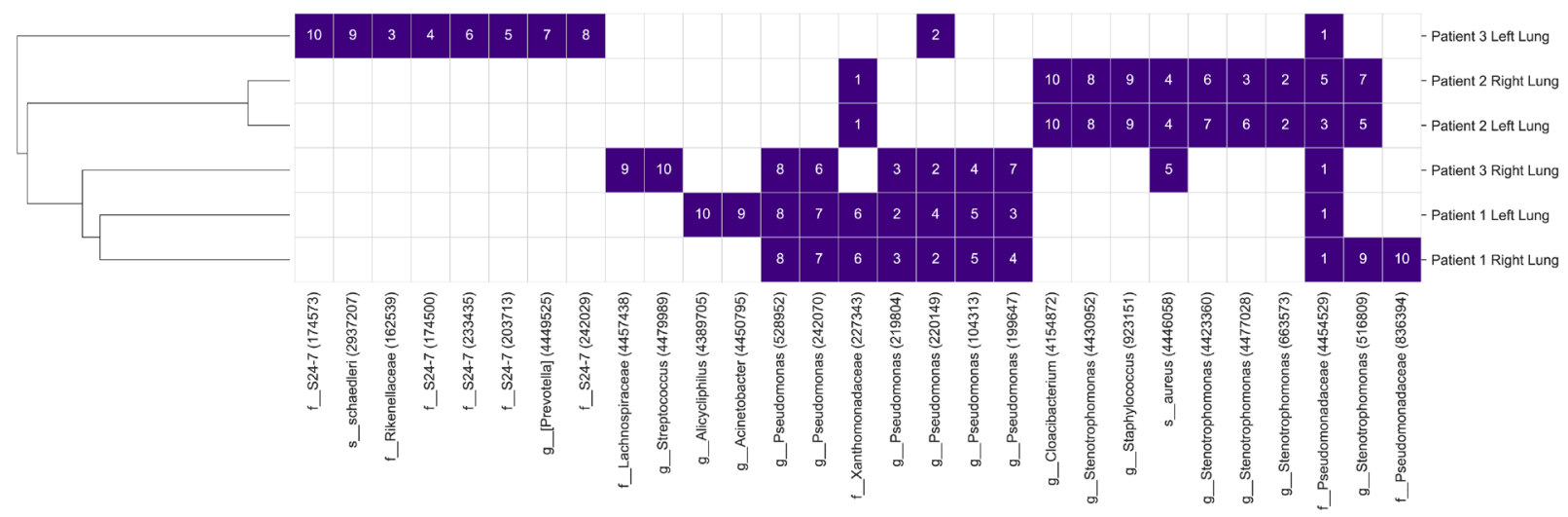

Supplementary Figure 3. Top 10 most abundant OTUs and their ranks (1 most abundant; 10 least abundant) according to host and sampling site. Columns represent OTUs and are labeled according to their taxonomic classification and their Greengenes identifier. Rows represent the sites and are hierarchically clustered based on the rank vectors.

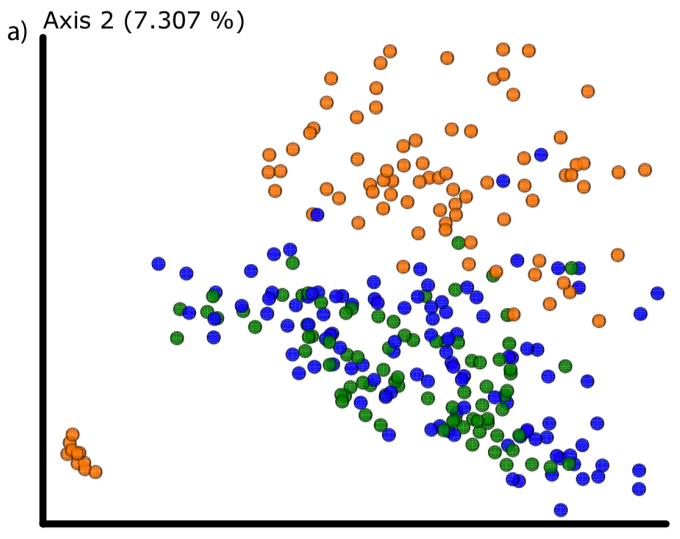

Axis $1(9.593 \%)$
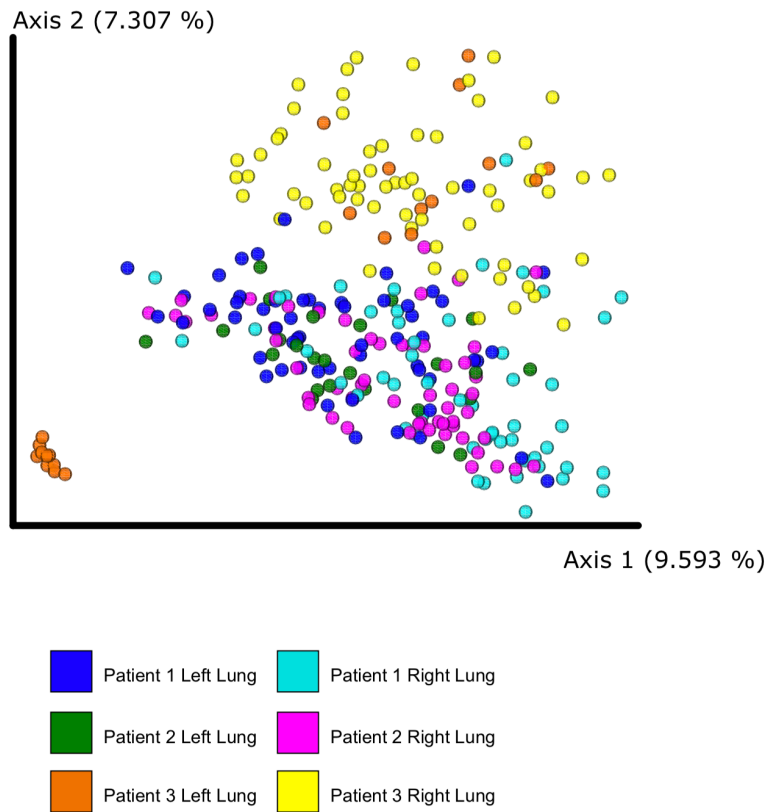

Supplementary Figure 4. Unweighted UniFrac PCoA plot of the $16 \mathrm{~S}$ data. a) Ordination colored by subject identifier. b) Ordination colored by subject identifier and sampling site. 


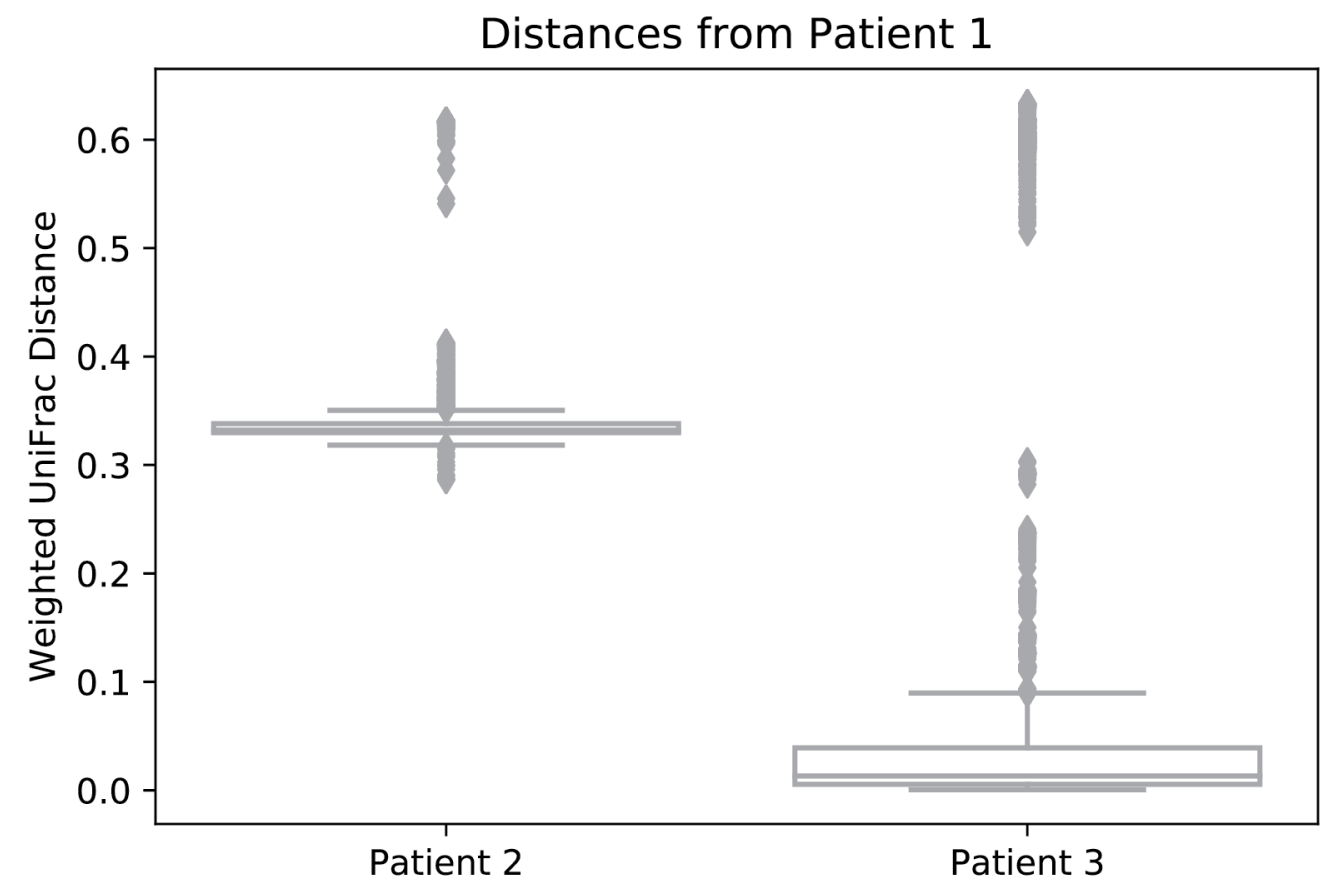

431

432 Supplementary Figure 5. Weighted UniFrac distances from Patient 1 to Patients 2 and 3. 
bioRxiv preprint doi: https://doi.org/101101/676148; this version posted June 192019 . The copyright holder for this preprint (which was not certified by peer review) is the author/funder, who has granted bioRxiv a license to display the preprint in perpetuity. It is made available under aCC-BY-NC-ND 4.0 International license.

a)

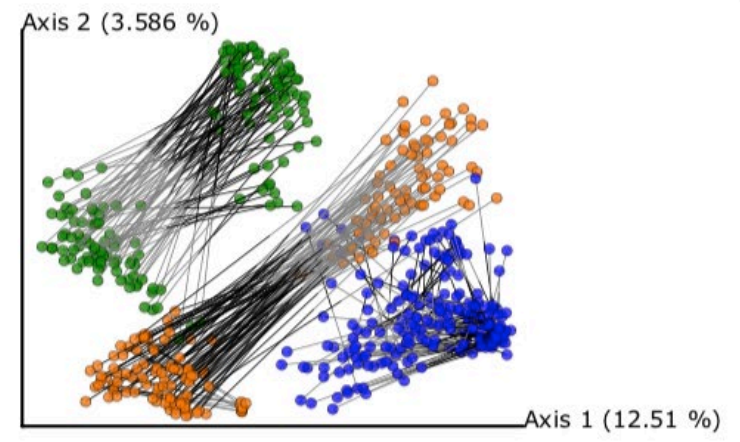

c)

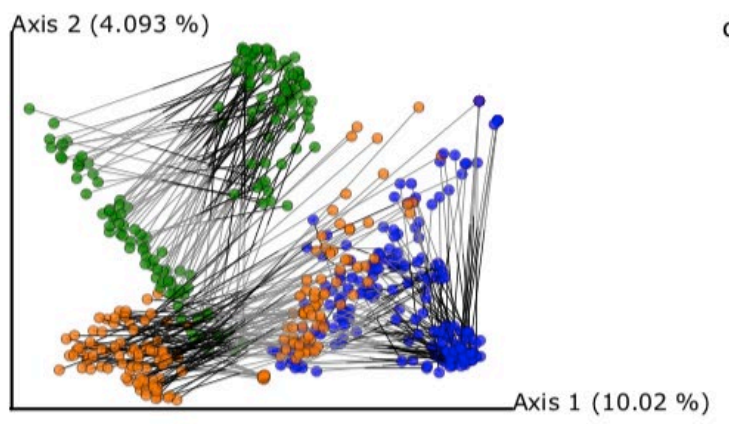

b)

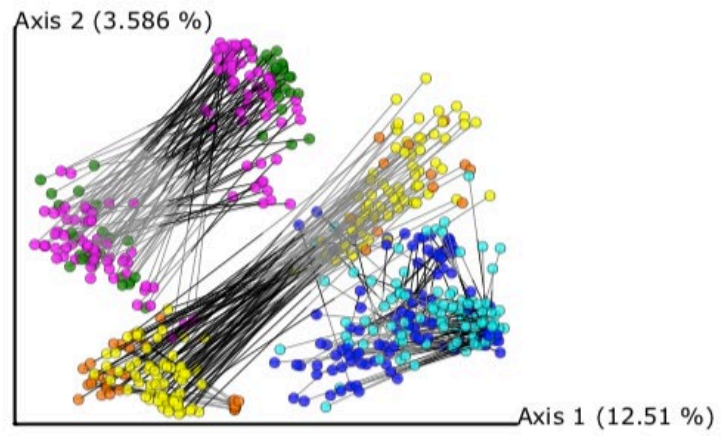

d)

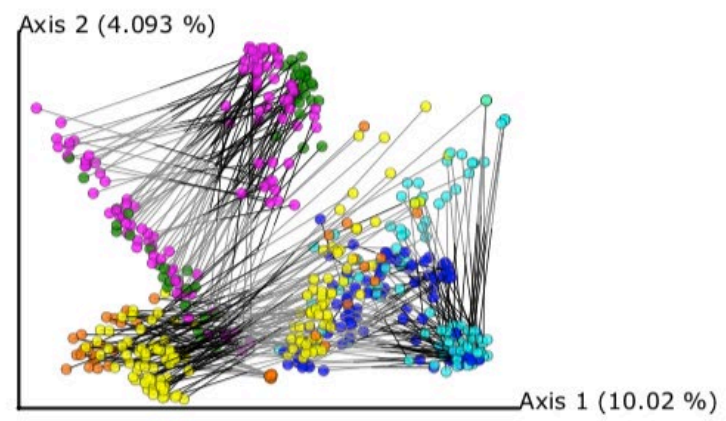

433

434

435

436

437
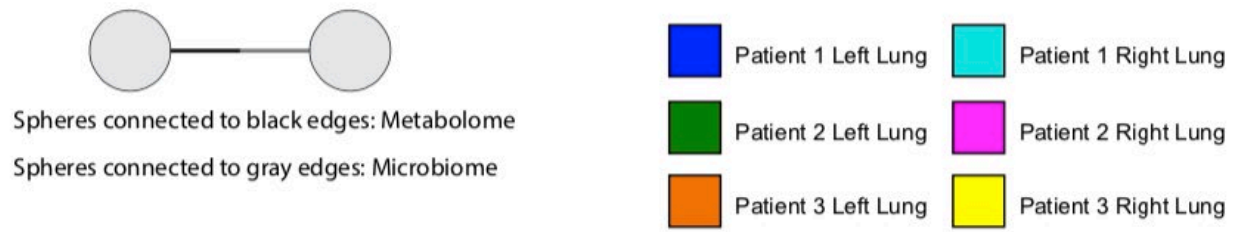

Supplementary Figure 6. Procrustes plots generated using principal component analysis of Canberra distance metric for untargeted metabolomics data and 16s rRNA sequencing data. a),b) - Emperor visualization plots of metabolomics and closed-reference picked OTUs. c),d) Emperor visualization plots of metabolomics and deblurred sOTUs. 
bioRxiv preprint doi: https://doi org/101101/676148; this version posted June 19, 2019. The copyright holder for this preprint (which was not certified by peer review) is the author/funder, who has granted bioRxiv a license to display the preprint in perpetuity. It is made available under aCC-BY-NC-ND 4.0 International license.

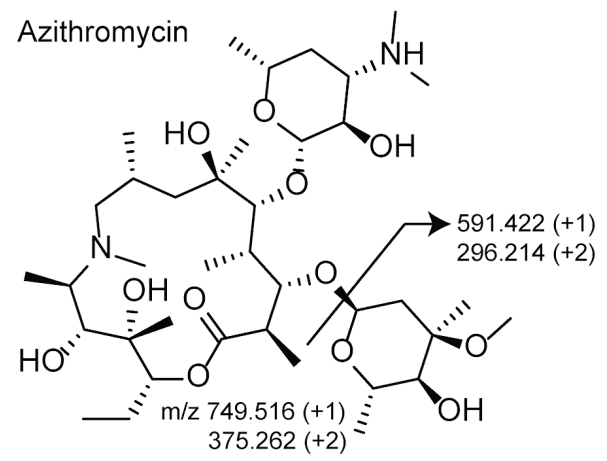<smiles>CNC1C[C@H](C)OC=C1O</smiles>

$\mathrm{m} / \mathrm{z} 158.118$<smiles>CNC1CCOCC1</smiles>

$\mathrm{m} / \mathrm{z} 116.107$

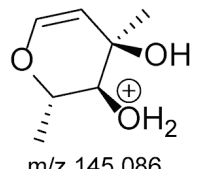

$\mathrm{m} / \mathrm{z} 145.086$<smiles>C[NH2+][C@H]1C[C@@H](C)OC=C1O</smiles>

$\mathrm{m} / \mathrm{z} 144.102$

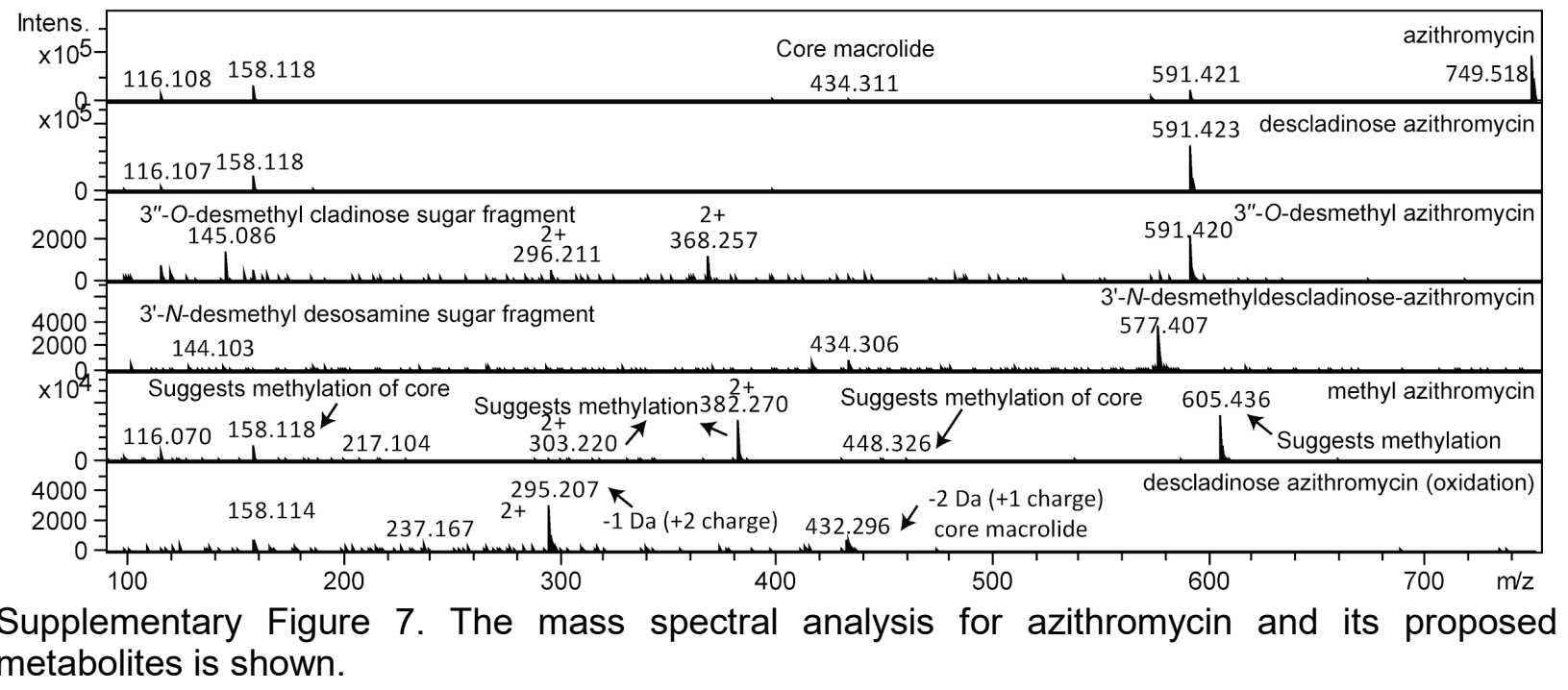


bioRxiv preprint doi: https://doi.org/101101/676148; this version posted June 19,2019. The copyright holder for this preprint (which was not aCC-BY-NC-ND 4.0 International license.

a)

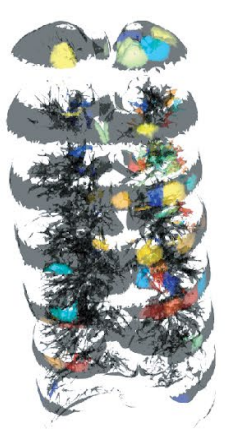

$-1-$

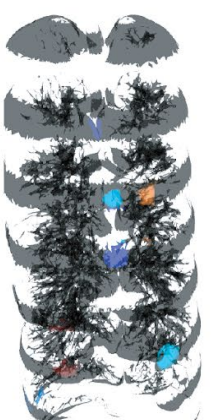

Jiperacillin metabolite $\mathrm{m} / \mathrm{z} 535$

Chlorhexidine

b)
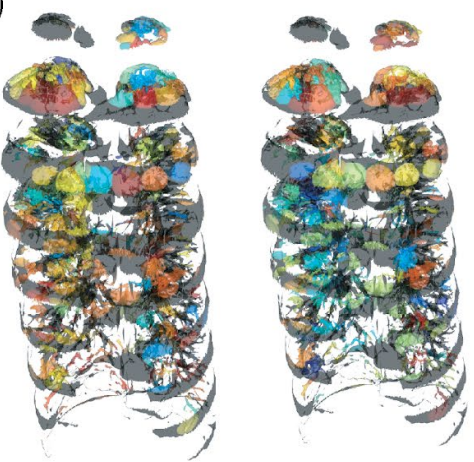

Trimethoprim

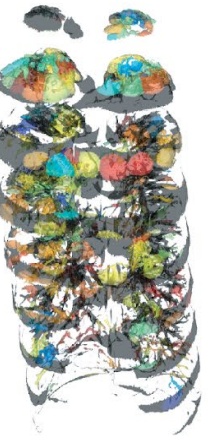

Sulfamethoxazole

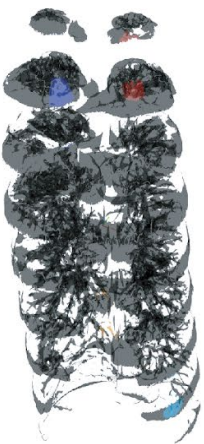

Chlorhexidine

c) $-1=$

Methylated-azithromycin

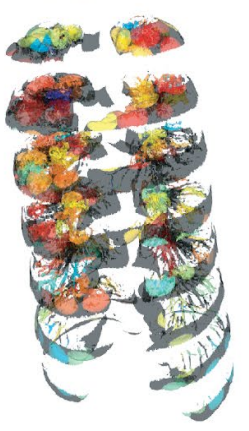

Ciprofloxacin

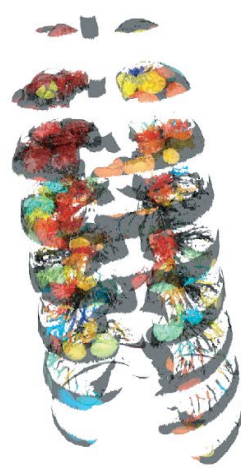

Ceftazidime

Supplementary Figure 8. Distribution of antibiotics and its metabolites in patients. a) patient 1, b) patient 2 and $\mathrm{c}$ ) patient 3 .
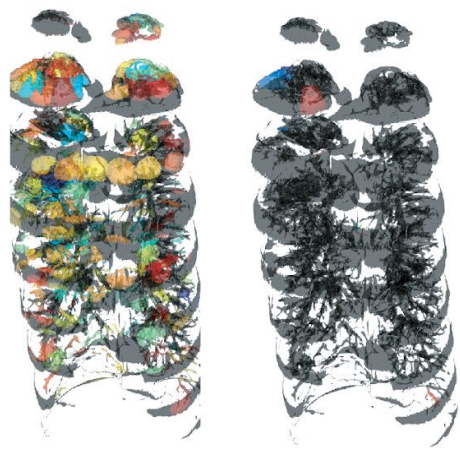

Sulfamethoxazole

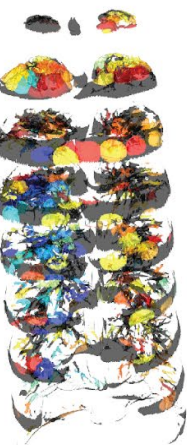

Linezolid

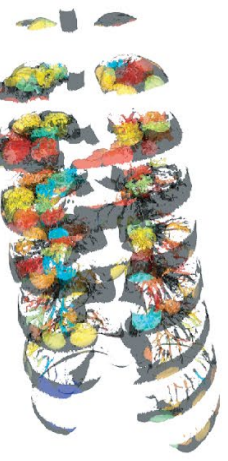

Methylated azithromycin
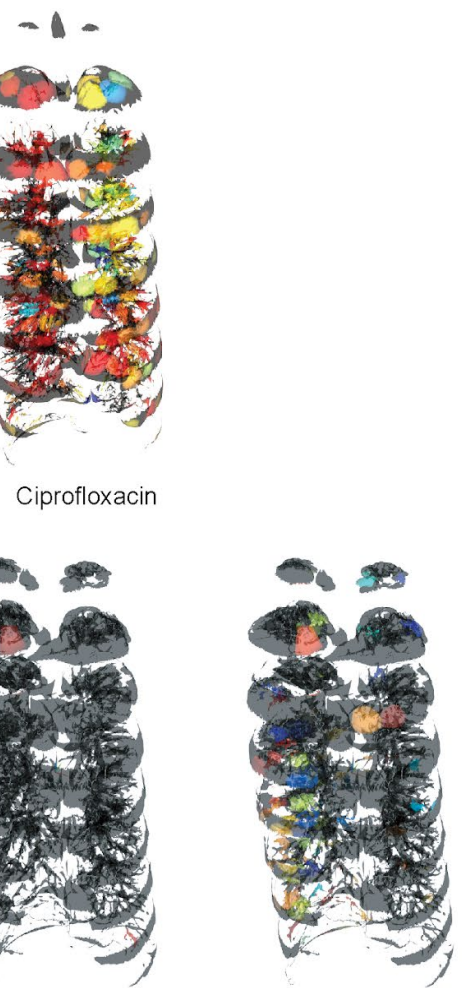

Sulfamethoxazole -metabolite $\mathrm{m} / \mathrm{z} 502$

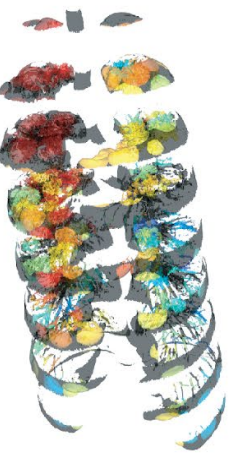

Piperacillin metabolite Piperacillin metabolitt $m / z 535$

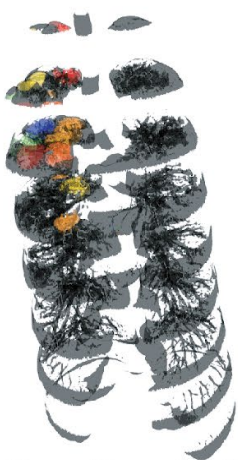

$\mathrm{m} / \mathrm{z} 507$ 
bioRxiv preprint doi: https://doi.org/10.1101/676148; this version posted June 19, 2019. The copyright holder for this preprint (which was not certified by peer review) is the author/funder, who has granted bioRxiv a license to display the preprint in perpetuity. It is made available under aCC-BY-NC-ND 4.0 International license.

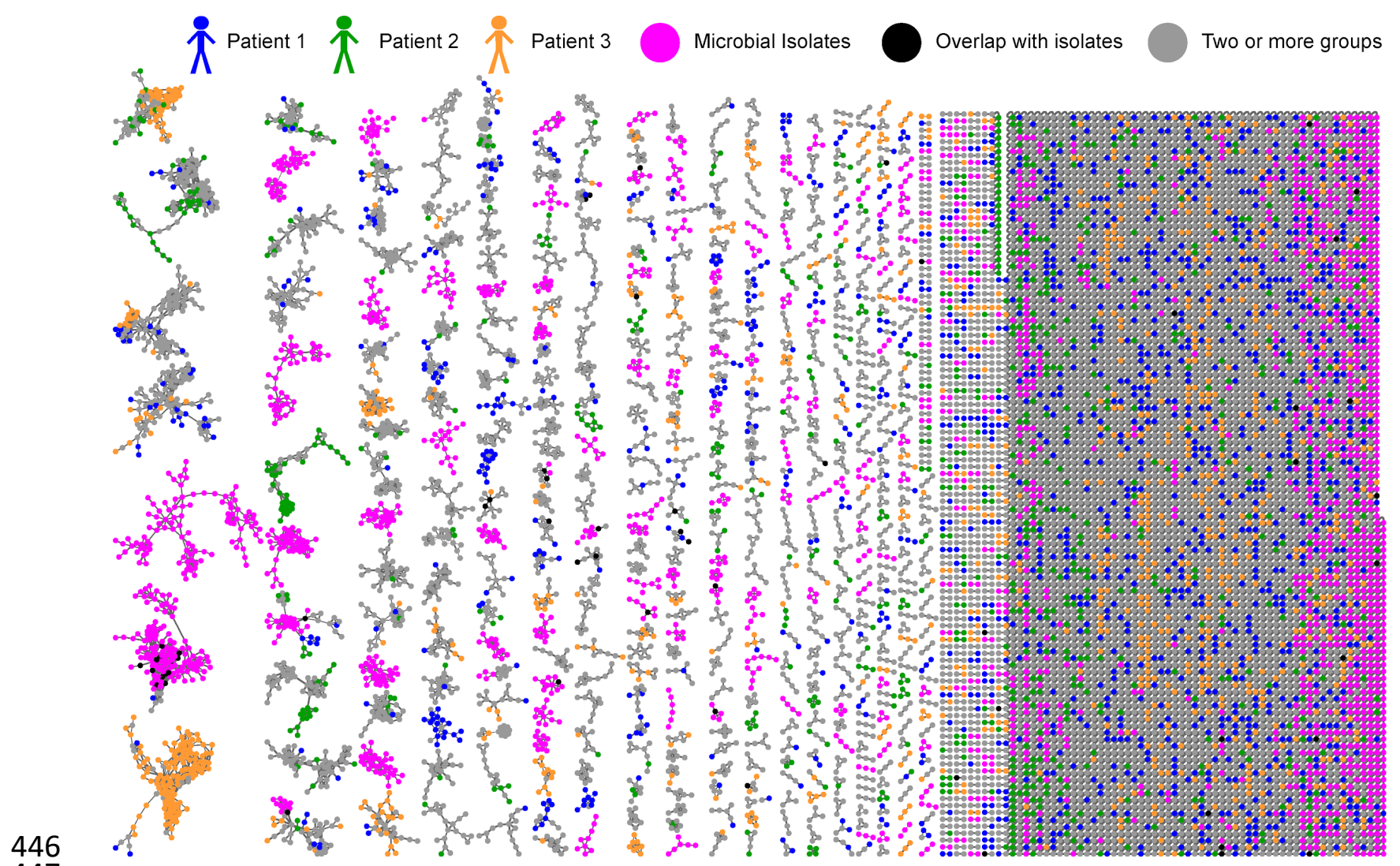

Supplementary Figure 9. Molecular network analysis of all six lungs from three patients and 449 microbial isolates. The molecular network is color coded by sample source. 


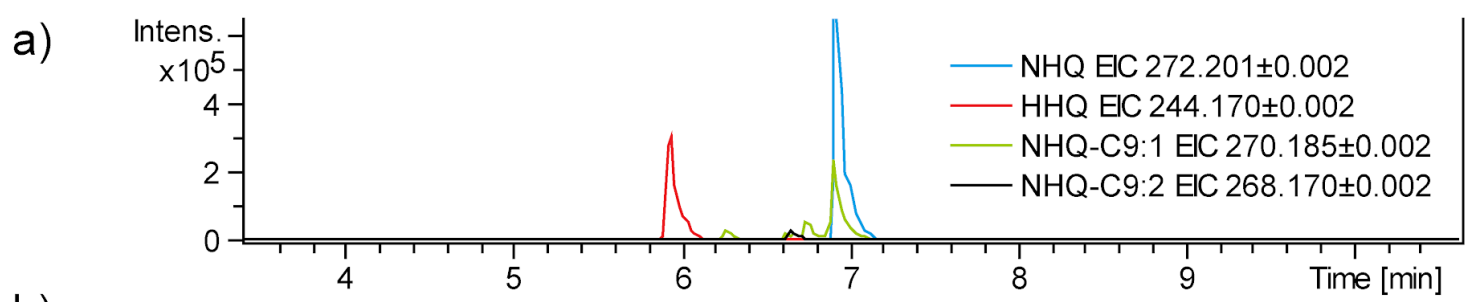

b)

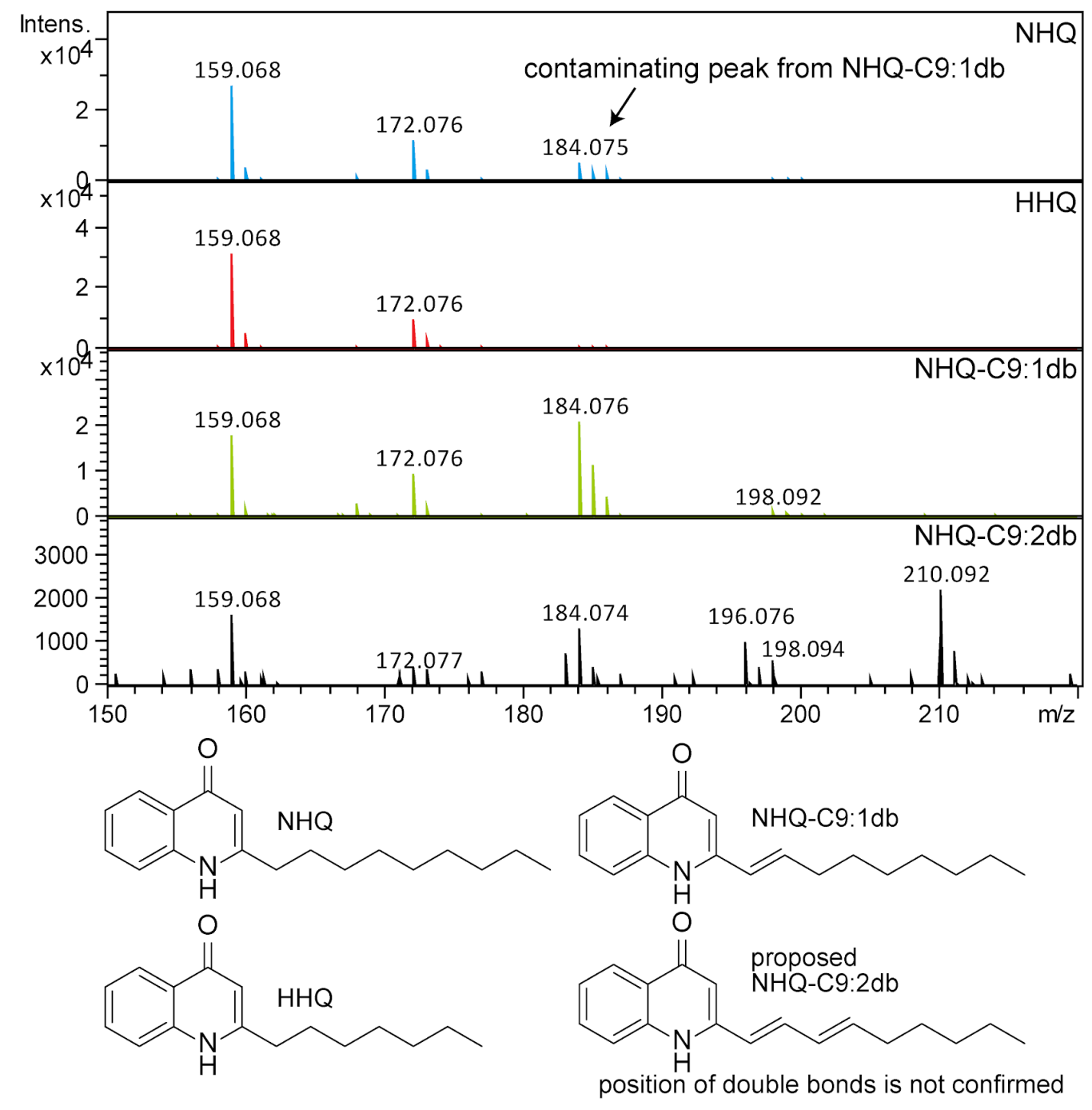

C)<smiles></smiles>

159.068<smiles>Oc1cc2c(c3ccccc13)CC=C2</smiles>

184.076<smiles></smiles>

172.076<smiles>Oc1cc2[n+](c3ccccc13)CCC=C2</smiles>

198.091<smiles></smiles>

196.076<smiles>Oc1cc[n+]2c(c1)C=CC=CC2</smiles>

210.091

Supplementary Figure 10. a) The extracted ion chromatograms (EIC), b) mass spectrum and structures of quinolones NHQ, HHQ, NHQ-C9:1db, NHQ-C9:2db (proposed) are shown. The position of additional double bond for NHQ-C9:2db is not known and is putatively drawn. c) The structures of fragments described previously in the literature for $\mathrm{NHQ}, \mathrm{HHQ}$ and $\mathrm{NHQ}-\mathrm{C} 9: 1 \mathrm{db}^{40}$ 
bioRxiv preprint doi: https://doi org/101101/676148; this version posted June 19,2019. The copyright holder for this preprint (which was not

and the proposed structures for fragments corresponding to NHQ-C9:2db are enclosed in brackets.

458

\section{P1}

a) Distribution of $\mathrm{NHQ}$

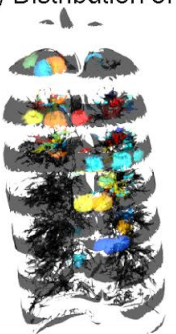

b) Distribution of NHQ-C9:1 db

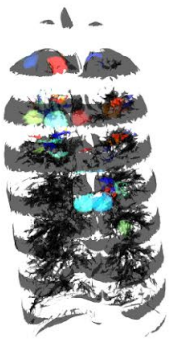

c) Distribution of $\mathrm{HHQ}$

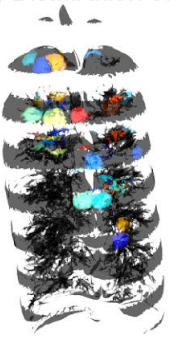

d) Distribution of UHQ-C11:1 db

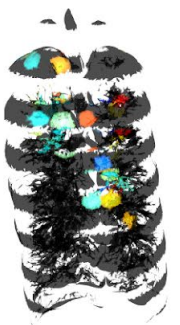

P3
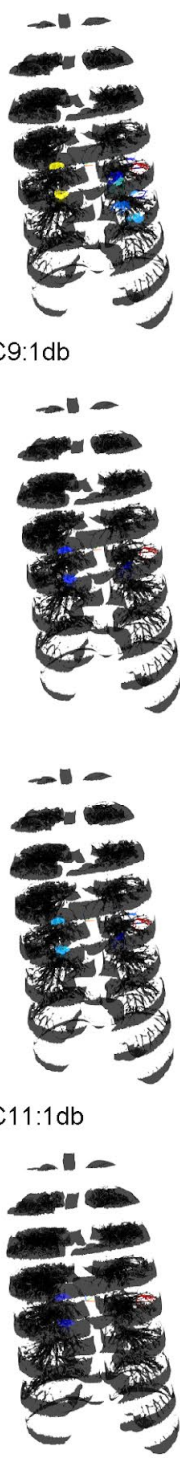

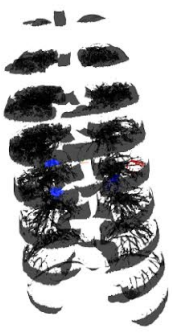

P1

f) Distribution of HHQ-C7:1db

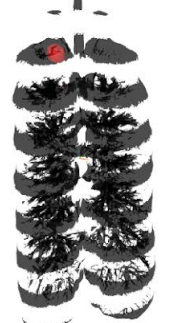

g) Distribution of NQNO-C9

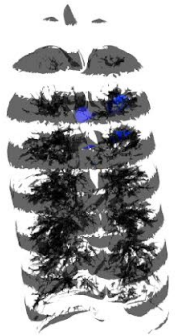

h) Distribution of $\mathrm{HHQ}-\mathrm{C} 10: 1 \mathrm{db}$

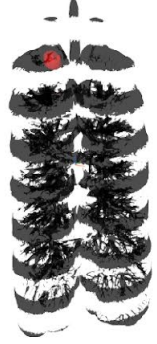

i) Distribution of NQNO-C9:1 db
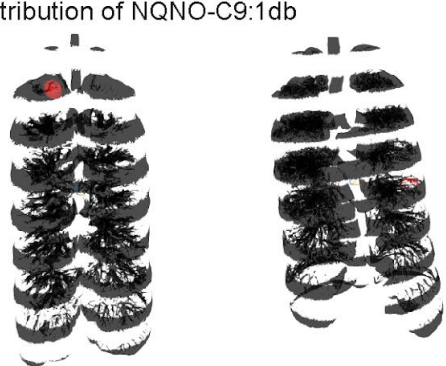

j) Distribution of HHQ-C13

P3
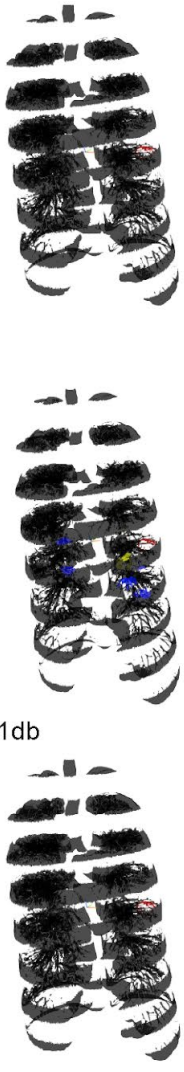

e) Distribution of $\mathrm{HHQ}-\mathrm{C} 13: 1 \mathrm{db}$

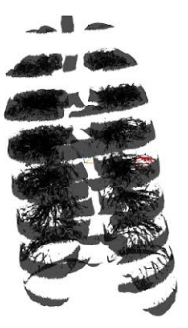

459

460 461
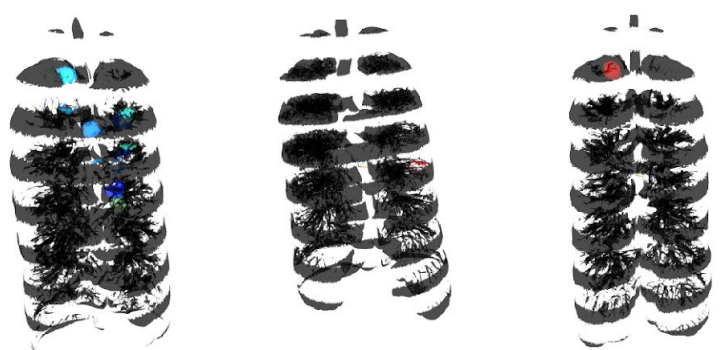

Supplementary Figure 11. Distribution of quinolones that are common to patients 1 and 3 .

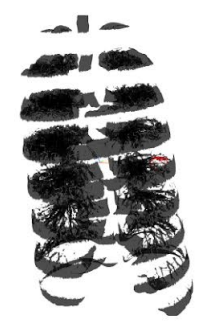

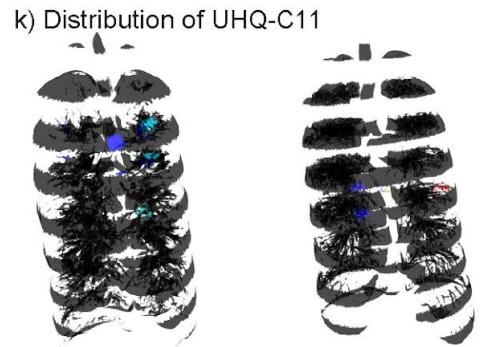

I) Distribution of $\mathrm{HHQ}-\mathrm{C} 8$

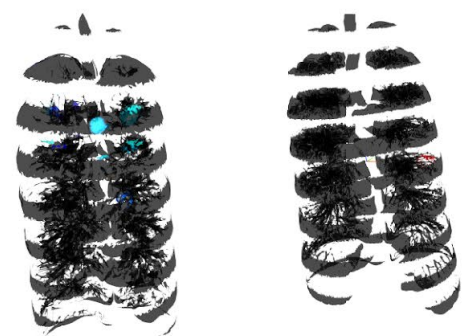

n) Distribution of $\mathrm{NHQ}-\mathrm{C} 9: 2 \mathrm{db}$

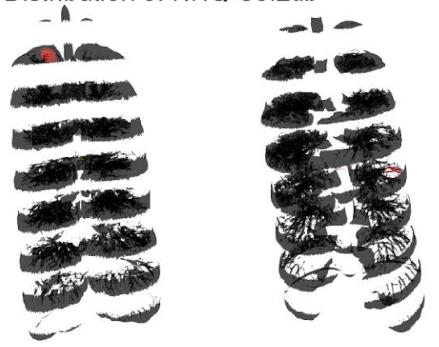

o) Distribution of HHQ-C12:1db HQNO-C11

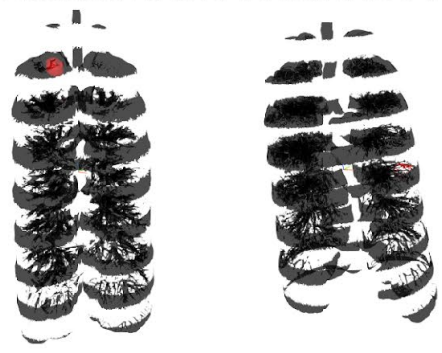

p) Distribution of HQNO-C11

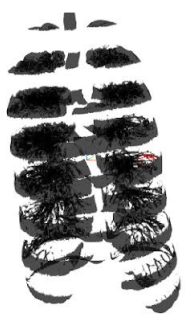




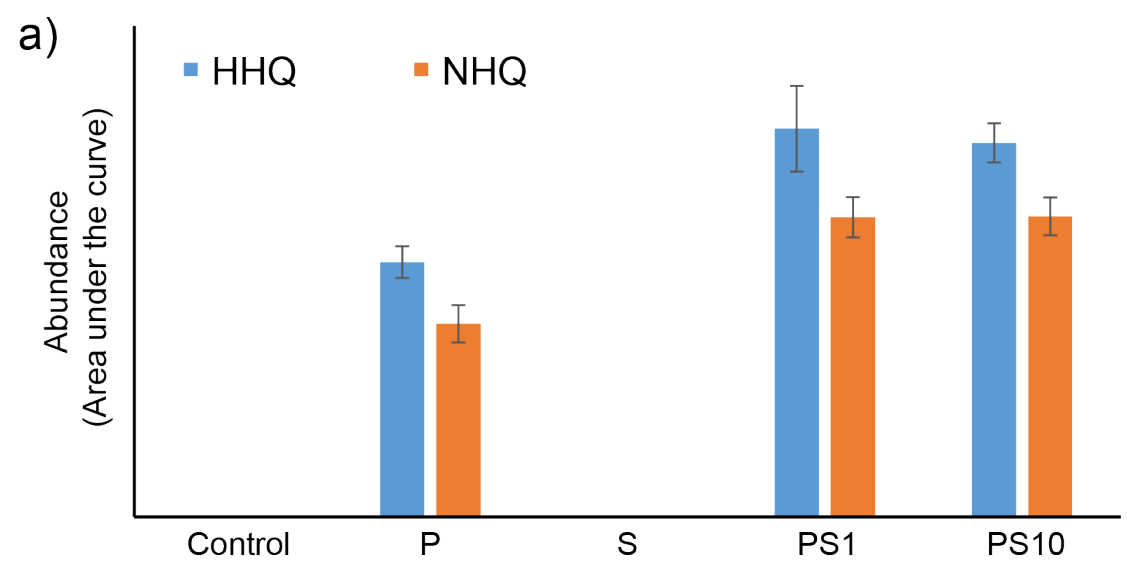

b)

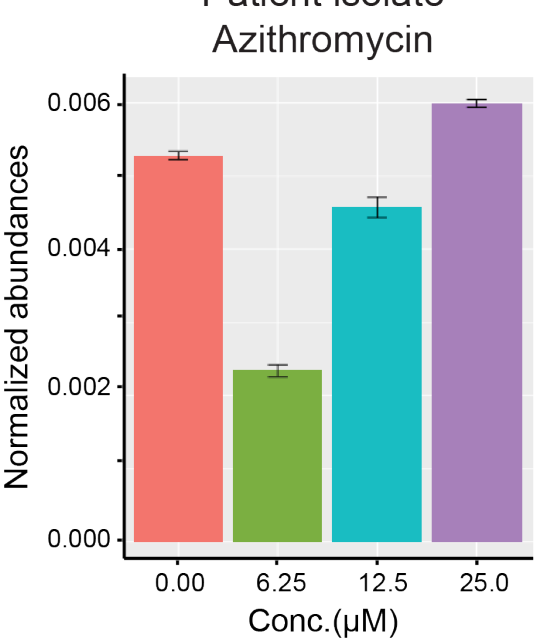

Patient isolate

Azithromycin

463

464

465

466

467

468

469

470

Supplementary Figure 12. Bar graphs of plots of area under the curves normalized to total ion current (error bars indicate standard deviation from mean values of three independent experiments $(n=3)$ ). a) Quinolone production by Pseudomonas aeruginosa in single culture and in mixed culture with Staphylococcus aureus is shown. PS1 represents cultures were mixed in the ratio 1:1 and PS10 represents a ratio of 1:10, where $S$. aureus culture was diluted 10 fold. b) Production of quinolone NHQ in response to antibiotic exposure by Pseudomonas aeruginosa isolated from patient 3 . Each color represent different concentrations of antibiotics. 
bioRxiv preprint doi: https://doi.org/10.1101/676148; this version posted June 19, 2019. The copyright holder for this preprint (which was not certified by peer review) is the author/funder, who has granted bioRxiv a license to display the preprint in perpetuity. It is made available under aCC-BY-NC-ND 4.0 International license.

471 Supplementary Table 1. Taxa summary table

\# Constructed from biom file

\#OTU ID

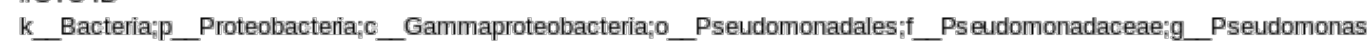

k_Bacteria; $p$ _Bacteroidetes; $\mathrm{C}$ __Flavobacteriia; $\mathrm{o}$ _ Flavobacteriales;f_[Weeksellaceae];g_Cloacibacterium

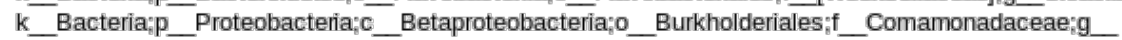

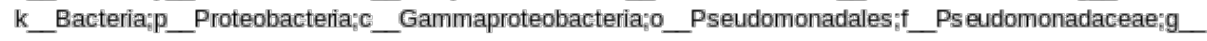

k_Bacteria; $\mathrm{P}$ __Proteobacteria; $\mathrm{C}$ _ Gammaproteobacteria; $\mathrm{O}$ _ Xanthomonadales; $\mathrm{f}$ __ Xanthomonadaceae;g_Stenotrophomonas

k__Bacteria; ___Proteobacteria;c__Gammaproteobacteria;o__Pseudomonadales; $f$ __Moraxellaceae;g_Acinetobacter

k_Bacteria;p_Firmicutes;c_Bacilli;o_Bacillales;f_Staphylococcaceae;g_Staphylococcus

k__Bacteria; ___Firmicutes;c_Bacilli;o__Bacillales;f__Bacillaceae;g_Bacillus

k__Bacteria; $p$ __Firmicutes;c_Bacilli;o_L_Lactobacillales; $;$ _Enterococcaceae;g_Enterococcus

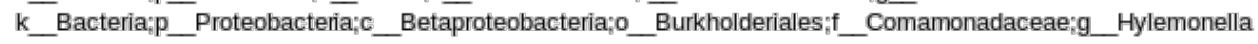

k_Bacteria; $\mathrm{p}$ __Proteobacteria; c _Betaproteobacteria; $\mathrm{o}$ _ Rhodocyclales; $;$ _R Rhodocyclaceae;g_Dechloromonas

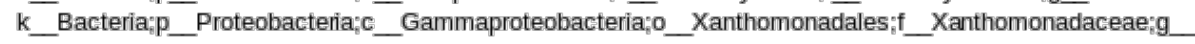

k_Bacteria; $;$ __Firmicutes;c_Clostridia;o_Clostridiales;f__Ruminococcaceae;g_Faecalibacterium

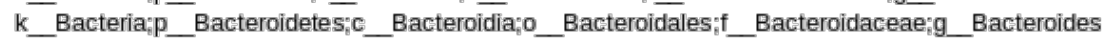

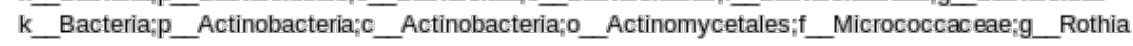

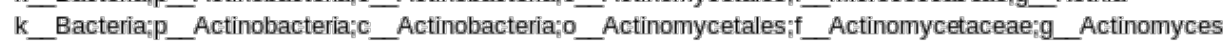

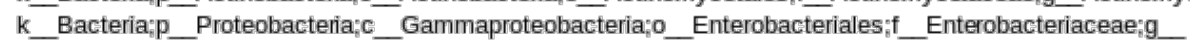

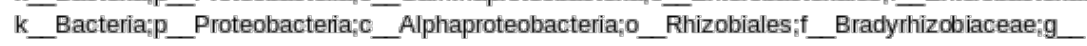

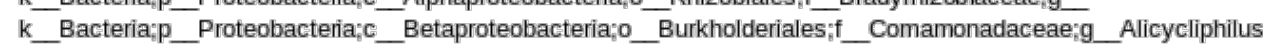

$\mathrm{k}$ __Bacteria; $\mathrm{p}$ _B Bacteroidetes;c_B_Bacteroidia;o_Bacteroidales:f_[Paraprevotellaceae]:g_[Prevotella]

k_Bacteria;p_Firmicutes;c_Bacilli;o_Lactobacillales;f_Streptococcaceae;g_Streptococcus

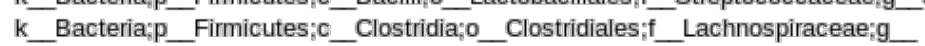

k_Bacteria; $p \_$_Proteobacteria; $c$ _Betaproteobacteria; $;$ __ Neisseriales:f_Neisseriaceae;g_Neisseria

k_Bacteria; ___Firmicutes;c_Bacilli; o__Gemellales;f___Gemellaceae;g__

k_Bacteria; $;$ ___Bacteroidetes;c_Bacteroidia;o_Bacteroidales; $f$ _Prevotellaceae;g_Prevotella

k_Bacteria;p_Firmicutes;c_Bacilli;o__Lactobacillales;f_Carnobacteriaceae;g_Granulicatella

k_Bacteria; $\mathrm{p}$ __Proteobacteria; $\mathrm{c}$ _ Gammaproteobacteria; $\mathrm{o}$ _Pasteurellales;f_Pasteurellaceae;g_Haemophilus

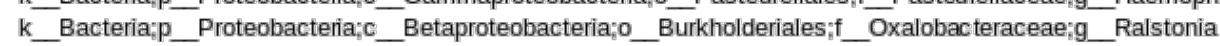

K__Bacteria; ___Firmicutes;c_Clostridia;o_Clostridiales;f_Veillonellaceae;g_Veillonella

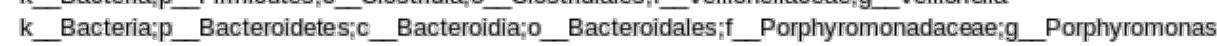

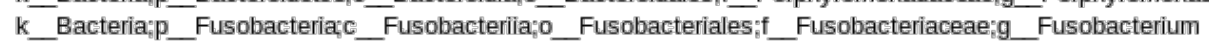

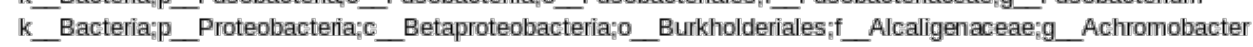

k_Bacteria; ;__Bacteroidetes;c__Bacteroidia;o_Bacteroidales;f_[Barnesiellaceae];g_

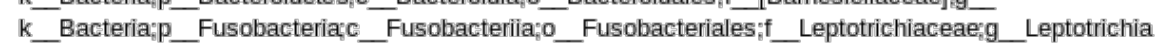

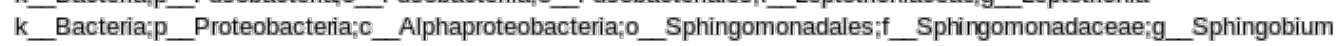

k_Bacteria; $\mathrm{p}$ __Proteobacteria; $\mathrm{c}$ _Betaproteobacteria; 0 __ Neisseriales;f_Neisseríaceae;g_Conchiformibius

k_Bacteria; $\mathrm{p}$ __Proteobacteria; $\mathrm{c}$ _Epsilonproteobacteria; ${ }_{0}$ __Campylobacterales;f_Campylobacteraceae;g_Campylobacter

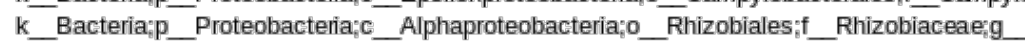

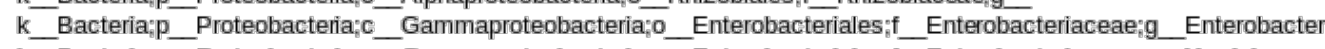

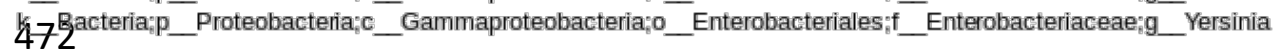

\begin{tabular}{|c|c|c|}
\hline patient_1 & patient_2 & batient_3 \\
\hline 0.987999611 & 0.0000134 & 0.978101853 \\
\hline 0.00275499 & 0.001671197 & 0.000108784 \\
\hline 0.002739072 & 0.001235305 & 0.0000858 \\
\hline 0.001915169 & 0.001952885 & 0.007403893 \\
\hline 0.001218315 & 0.915415264 & 0.000047 \\
\hline 0.001097751 & 0.000449253 & 0.0000796 \\
\hline 0.000685652 & 0.012052438 & 0.011663445 \\
\hline 0.00037761 & 0.0000507 & 0.0000301 \\
\hline 0.000318949 & 0.00000668 & 0 \\
\hline 0.000273553 & 0.000110226 & 0 \\
\hline 0.000243486 & 0.000118019 & 0 \\
\hline 0.000239949 & 0.062973295 & 0.0000707 \\
\hline 0.0000607 & 0.000868444 & 0.0000801 \\
\hline 0.0000528 & 0.003076851 & 0.0000879 \\
\hline 0.00000973 & 0.00000111 & 0.000120101 \\
\hline 0.00000855 & 0.00000111 & 0.0000271 \\
\hline 0.00000265 & 0.00000334 & 0.0000351 \\
\hline 0.000000884 & 0 & 0.000337207 \\
\hline 0.00000059 & 0 & 0.0000825 \\
\hline 0 & 0.000000557 & 0.00000508 \\
\hline 0 & 0 & 0.000698204 \\
\hline 0 & 0 & 0.000280506 \\
\hline 0 & 0 & 0.000117676 \\
\hline 0 & 0 & 0.0000984 \\
\hline 0 & 0 & 0.0000903 \\
\hline 0 & 0 & 0.0000762 \\
\hline 0 & 0 & 0.0000654 \\
\hline 0 & 0 & 0.0000619 \\
\hline 0 & 0 & 0.0000462 \\
\hline 0 & 0 & 0.0000385 \\
\hline 0 & 0 & 0.0000182 \\
\hline 0 & 0 & 0.0000117 \\
\hline 0 & 0 & 0.00000901 \\
\hline 0 & 0 & 0.00000658 \\
\hline 0 & 0 & 0.00000612 \\
\hline 0 & 0 & 0.00000404 \\
\hline 0 & 0 & 0.00000381 \\
\hline 0 & 0 & 0.00000104 \\
\hline 0 & 0 & 0 \\
\hline 0 & 0 & 0 \\
\hline
\end{tabular}

473

474 
475 Supplementary Table 2. Percentage of shared mass spectrometry features between left and 476 right lungs of all patients. This percentage was calculated by taking the features that overlap in 477 comparison to the total number of features associated with each pair of lungs.

478

\begin{tabular}{|c|c|c|c|c|c|c|c|}
\hline \multicolumn{2}{|c}{} & P1 & P1 & P2 & P2 & P3 & P3 \\
\cline { 3 - 8 } & Left & Right & Left & Right & Left & Right \\
\hline P1 & Left & 100 & 88.02 & 75.71 & 76.92 & 70.46 & 72.7 \\
\hline P1 & Right & 88.02 & 100 & 71.67 & 72.39 & 74.01 & 76.73 \\
\hline P2 & Left & 75.71 & 71.67 & 100 & 89.46 & 67.91 & 70.54 \\
\hline P2 & Right & 76.92 & 72.39 & 89.46 & 100 & 70.35 & 72.74 \\
\hline P3 & Left & 70.46 & 74.01 & 67.91 & 70.35 & 100 & 91.56 \\
\hline P3 & Right & 72.7 & 76.73 & 70.54 & 72.74 & 91.56 & 100 \\
\hline
\end{tabular}

479

480 


\section{Materials and Methods.}

482 Tissue collection and processing. To map the microbiome and metabolome of explanted

483 lungs in $3 \mathrm{D}$, the lungs of three patients were obtained in close coordination with the patient's

484 physician and the surgical team. This work was approved by the University of California

485 Institutional Review Board (project \#081500) and informed consents were obtained prior to

486 tissue collection. The CFTR mutation in Patient 1 was dF508/G551D with no clinical diabetes, in

487 patient 2 was $\mathrm{dF} 508 / 3120+1 \mathrm{G}>\mathrm{A}$ with observed clinical diabetes and in patient 3 was

488 dF508/dF508 with observed clinical diabetes. The general workflow for tissue sectioning is

489 described previously (16). Briefly, both the right and left lungs for subject 1,2 , and 3 were

490 collected. The tissue sectioning was performed at the hospital under the guidance of a

491 pathologist. The lungs were first sliced horizontally. The anatomical orientation of each slice was

492 recorded. Every alternate slice starting from apex of the lung was further sub-sectioned into

493 small sections $1-2 \mathrm{~cm}^{3}$ in size maintaining the recorded orientation. Each of the sub-sectioned

494 tissue pieces were swabbed with sterile soft foam swabs moistened with Tris-EDTA, pH 7.4.

495 The swabs were stored in 96-well bead plate provided in the PowerSoilß-htp 96 Well Soil DNA

496 Isolation Kit. The plate was placed on dry ice prior and during the collection. The individual

497 tissue pieces were stored in glass jars placed on dry ice. The samples were kept frozen at -80

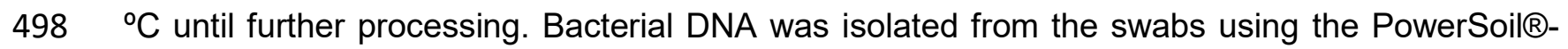

499 htp 96 Well Soil DNA Isolation Kit following the manufacturer's instructions and was subjected to

500 prokaryotic ribosomal 16S rRNA-based sequencing using the standardized Earth Microbiome

501 Protocol (http://www.earthmicrobiome.org/emp-standard-protocols/). Amplicons were cleaned,

502 pooled and then sequenced on an Illumina MiSeq. Because lungs were obtained at different

503 times, the sequences analyzed for this study were obtained from a total of two individual

504 sequencing runs (sequencing run 1: patients 1-2, sequencing run 2: patient 3 ). The sequencing

505 runs were performed at the University of California San Diego Institute for Genomic Medicine

506 Center. For untargeted metabolomics analysis, the tissue sections were weighed and extracted 
507 with $1 \mathrm{~mL} / \mathrm{g}$ of tissue with a 2:2:1 mixture of ethyl acetate, methanol and water. An aliquot of 150

$508 \mu \mathrm{L}$ of the extract was dried for each tissue section and analyzed by MS.

510 MS data acquisition. The tissue extracts and extracts of bacterial isolates from the subjects

511 cultured on sheep blood agar and MacConkey agar were resuspended in $80 \%$ methanol

512 containing $1 \mu \mathrm{M}$ sulfadimethoxine and analyzed with a UltiMate 3000 UHPLC system (Thermo

513 Scientific) using a Kinetex ${ }^{\mathrm{TM}} 1.7 \mu \mathrm{m}$ C18 reversed phase UHPLC column $(50 \times 2.1 \mathrm{~mm})$ and

514 Maxis Q-TOF mass spectrometer (Bruker Daltonics) equipped with ESI source. The column was

515 equilibrated with $2 \%$ solvent B (98\% acetonitrile, $0.1 \%$ formic acid in LC-MS grade water with

516 solvent $A$ as $0.1 \%$ formic acid in water) for $1 \mathrm{~min}$, followed by a linear gradient from $2 \%$ B to

$517100 \%$ B in $10 \mathrm{~min}$, held at $100 \%$ B for $2.5 \mathrm{~min}$. A small wash segment was employed to wash

518 the column (100\% B for $0.5 \mathrm{~min}, 100 \%-10 \% \mathrm{~B}$ in $0.5 \mathrm{~min}$ ) following which the column was kept

519 at $2 \% \mathrm{~B}$ for $\mathrm{A}$ min at a flow rate of $0.5 \mathrm{~mL} / \mathrm{min}$ throughout the run. MS spectra were acquired in

520 positive ion mode in the range of $50-2000 \mathrm{~m} / \mathrm{z}$. A mixture of $10 \mu \mathrm{g} / \mathrm{mL}$ of each sulfamethazine,

521 sulfamethizole, sulfachloropyridazine, sulfadimethoxine, amitriptyline, and coumarin-314 was

522 run after every eight injections for quality control. An external calibration with ESI-L Low

523 Concentration Tuning Mix (Agilent technologies) was performed prior to data collection and

524 internal calibrant Hexakis $(1 \mathrm{H}, 1 \mathrm{H}, 3 \mathrm{H}$-tertrafluoropropoxy)phosphazene was used throughout the

525 runs. The capillary voltage of $4500 \mathrm{~V}$, nebulizer gas pressure (nitrogen) of 2 bar, ion source

526 temperature of $200{ }^{\circ} \mathrm{C}$, dry gas flow of $9 \mathrm{~L} /$ min source temperature, spectral rate of $3 \mathrm{~Hz}$ for $\mathrm{MS}^{1}$

527 and $10 \mathrm{~Hz}$ for $\mathrm{MS}^{2}$ was used. For acquiring MS/MS fragmentation, the 10 most intense ions per

$528 \mathrm{MS}^{1}$ were selected and collision induced dissociation energy given in Table 1 was used. Basic

529 stepping function was used to fragment ions at $50 \%$ and $125 \%$ of the CID calculated for each

$530 \mathrm{~m} / \mathrm{z}$ from Table 1 with timing of $50 \%$ for each step. Similarly, basic stepping of collision RF of

531550 and $800 \mathrm{Vpp}$ with a timing of $50 \%$ for each step and transfer time stepping of 57 and $90 \mu \mathrm{s}$

532 with a timing of $50 \%$ for each step was employed. MS/MS active exclusion parameter was set to 
5333 and released after 30 seconds. The mass of internal calibrant was excluded from the MS/MS

534 list using a mass range of $m / z$ 921.5-924.5. The data was deposited in the online repository

535 namely MassIVE and is available under the id MSV000079652 and MSV000079398.

536 The microbial isolates of Pseudomonas aeruginosa, Staphylococcus aureus and

537 Stenotrophomonas maltophila from the patients were obtained from the Center of Advanced

538 Clinical Medicine, UC San Diego. The culturing of the isolates and the extractions were

539 performed as described previously (16). The MS data was collected using the same conditions

540 as described above for lung tissue.

541

542 LC-MS/MS data analysis. All mzXML files were cropped with an $\mathrm{m} / \mathrm{z}$ range of 50.00 to

$5432,000.00 \mathrm{Da}$ and RT range of $0.5-18.5 \mathrm{~min}$. Feature extraction was performed using MZmine2

544 (http://mzmine.sourceforge.net/) with a signal height threshold of 5.0e3 (41). The mass

545 tolerance was set to $10 \mathrm{ppm}$, and the maximum allowed retention time deviation was set to 0.01

546 min. For chromatographic deconvolution, the local minimum search algorithm was used with a

547 minimum relative peak height of $1 \%$ and a minimum retention time range of $0.01 \mathrm{~min}$. The

548 maximum peak width was set to $1 \mathrm{~min}$. After isotope peak removal, the peak lists of all samples

549 were aligned with the above-mentioned retention time and mass tolerances. After the creation of

550 a feature matrix containing the feature retention times and the exact mass and peak areas of

551 the corresponding extracted ion chromatograms, the metadata of the samples were added. The

552 signal intensities of the features were normalized (probabilistic quotient normalization [PQN])

$553(42)$.

554

555 Statistical analysis was carried out as follows: QIIME 1.9.1 was used to perform principal556 coordinate analysis (PCoA) (beta_diversity.py, a Canberra distance metric in Adkins form). The 557 PCoA plots were visualized in EMPeror (28). 
559 Molecular networking. The molecular network was created using the online workflow at GNPS

560 platform. The data was then clustered with MS-Cluster with a parent mass tolerance of $0.1 \mathrm{Da}$

561 and a MS/MS fragment ion tolerance of 0.1 Da to create consensus spectra. Further, consensus

562 spectra that contained less than 3 spectra were discarded. A network was then created where

563 edges were filtered to have a cosine score above 0.7 and more than 4 matched peaks. The

564 edges between two nodes were kept in the network if and only if each of the nodes appeared in

565 each other's respective top 10 most similar nodes. The spectra in the network were then

566 searched against GNPS's spectral libraries. All matches kept between network spectra and

567 library spectra were required to have a score above 0.7 and at least 4 matched peaks. The

568 molecular networks and the parameters used are available at the links below:

569 The molecular network and parameters for the patient data are available at:

570 https://gnps.ucsd.edu/ProteoSAFe/status.jsp?task=6f92a21af31d4569bcdb3cce803c600c

571 The molecular network and parameters for the patient data and data acquired on cultured

572 microbial isolates are available at:

573 https://gnps.ucsd.edu/ProteoSAFe/status.jsp?task=45d70e56faae4081 bbba1f7a9ce38019

574 In total, 1776 of the nodes were annotated $(7.8 \%)$ which is higher than the typical rate of

575 annotations of $1.8 \%$ annotated in an untargeted metabolomics experiment (30). This is likely

576 because many of the reference MS/MS libraries in the public domain are populated from studies

577 of human samples and also contain most of therapeutics used in the clinic. The error rate of

578 these annotations have been assessed by the GNPS community; with the scoring settings used

579 to obtain the annotations, $1 \%$ of which are deemed incorrect, for $4 \%$ not enough information is

580 available and $4 \%$ could be an isomer or correct, while $91 \%$ is presumed correct (30).

581

582 16S rRNA gene analysis. As described above, sequences were obtained over the course of

583 two months through two independent sequencing runs. The samples for patient 1 and patient 2

584 were sequenced in one batch and samples for patient 3 were sequenced separately. Each set 
585 of sequences were processed and analyzed using Qiita (43). First, the sequencing runs were

586 quality trimmed and filtered using default parameters, resulting in 15,629,914 sequences with a

587 mean length of 150 nucleotides. Next, trimmed sequences (at 150 nucleotides) were clustered

588 into operational taxonomic units (OTUs) using the closed reference OTU picking method at $97 \%$

589 sequence similarity. UCLUST was the underlying clustering algorithm and Greengenes (August

5902013 release) was the reference database used (44). This resulted in 340 samples with a mean

591 of 25,078 sequences per sample. After rarefaction at 3,369 sequences per sample 277 samples

592 were used for downstream analyses, including the creation of taxonomy summaries and in the

593 calculation of the UniFrac and Canberra (Adkins form) distances. The most abundant OTU for

594 patient 2 was identified as unclassified genus in the family Xanthomonadaceae. BLAST analysis

595 of the sequence corresponding to this OTU from patient 2 revealed that it belongs to the genus

596 Stenotrophomonas. As controls, a total of 49 wells (either containing blank swab or empty well)

597 were interleaved between each of the two sampling sites (left and right lung) of the three

598 subjects. The vast majority of the samples (72\%) yielded zero sequences. The remaining 14

599 samples had a non-zero amount of sequences. Of these, 7 samples were represented by under

6004 sequences, a negligible amount compared to the 3,500 sequences per sample used for

601 analysis. And the last 7 samples were represented by over 6,000 sequences each. Although the

602 last sample set was processed without any DNA, the well-to-well contamination during the DNA

603 extraction step yielded these sequences. We removed these samples since the DNA is

604 biological and not representative of a type of actionable contamination $(45,46)$.

605

606 For statistical analysis, QIIME2 (47) was used to perform PCoA (Canberra (in Adkins form), 607 weighted, and unweighted UniFrac distances (weighted UniFrac distances (48)) and Procrustes 608 analysis with metabolomics data. The PCOA and Procrustes plots were visualized in EMPeror 609 (28). The Mantel test was used to calculate $r^{2}$ scores between mass spectrometry and for both 
610 closed-reference and deblur 16S rRNA gene analysis data using scikit-bio's 0.5.5 Mantel's test

611 implementation.

612 3D lung model generation and visualization. The procedure for creation and visualization of

613 3D models has been previously described (16). Briefly, the CT- scan images obtained from the

614 radiology department at the Hillcrest hospital in San Diego were combined to create a 3D lung

615 model and exported in the .stl format using InVesalius 3.0. The extraneous pixels corresponding

616 to the chest and back of each model were manually deleted using the 3D modelling software

617 Geomagic Wrap. The relative abundances of detected microbes and molecules were plotted on

618 to these models using a modified version of the 'ili software available at

619 http://mingwangbeta.ucsd.edu/public/ili/ $(16,49)$.

620

621 Data availability statement. All data presented in this manuscript is publically available. The

622 metabolomics data was deposited in the online repository namely MassIVE and is available

623 under the id MSV000079652 and MSV000079398. The molecular network analysis and

624 parameters for the patient data are available at:

625 https://gnps.ucsd.edu/ProteoSAFe/status.jsp?task=6f92a21af31d4569bcdb3cce803c600c

626 The molecular network analysis and parameters for the patient data and data acquired on

627 cultured microbial isolates are available at:

628 https://gnps.ucsd.edu/ProteoSAFe/status.jsp?task=45d70e56faae4081bbba1f7a9ce38019

629 All raw and processed $16 \mathrm{~S}$ amplicon sequencing data and metadata are available with Qiita

630 study identification 10169 and as EBI study with accession no. ERP110498.

631 All figures in this manuscript have associated raw data, which is available through above 632 described accession numbers. 
634 Code availability Statement. The code for 3D mapping via the browser tool github iili is

635 available at 'https://github.com/mwang87/ili and the tool is accessed via the link

636 http://mingwangbeta.ucsd.edu/public/ili/

637 Acknowledgements. We thank Amnon Amir from the Cancer Research Institute, Sheba

638 Medical Center, Israel for assisting in the analysis of deblurred data used in this article. 


\section{References}

640 1. Laxminarayan R, Duse A, Wattal C, Zaidi AK, Wertheim HF, Sumpradit N, Vlieghe E, Hara GL, Gould IM, Goossens H, Greko C, So AD, Bigdeli M, Tomson G, Woodhouse W, Ombaka E, Peralta AQ, Qamar FN, Mir F, Kariuki S, Bhutta ZA, Coates A, Bergstrom R, Wright GD, Brown ED, Cars O. 2013. Antibiotic resistance-the need for global solutions. Lancet Infect Dis 13:1057-98.

2. Shallcross LJ, Davies DS. 2014. Antibiotic overuse: a key driver of antimicrobial resistance. Br J Gen Pract 64:604-5.

3. Ventola CL. 2015. The antibiotic resistance crisis: part 1: causes and threats. P T 40:277-

83.

4. Mollenkopf DF, Stull JW, Mathys DA, Bowman AS, Feicht SM, Grooters SV, Daniels JB, Wittum TE. 2017. Carbapenemase-Producing Enterobacteriaceae Recovered from the Environment of a Swine Farrow-to-Finish Operation in the United States. Antimicrob Agents Chemother 61:e01298-16.

5. de Kraker ME, Stewardson AJ, Harbarth S. 2016. Will 10 Million People Die a Year due to Antimicrobial Resistance by 2050? PLoS Med 13:e1002184.

6. Woolhouse M, Waugh C, Perry MR, Nair H. 2016. Global disease burden due to antibiotic resistance - state of the evidence. J Glob Health 6:010306.

7. Venkataraman A, Rosenbaum MA, Werner JJ, Winans SC, Angenent LT. 2014. Metabolite transfer with the fermentation product 2,3-butanediol enhances virulence by Pseudomonas aeruginosa. ISME J 8:1210-20.

8. Nougayrede JP, Homburg S, Taieb F, Boury M, Brzuszkiewicz E, Gottschalk G, Buchrieser C, Hacker J, Dobrindt U, Oswald E. 2006. Escherichia coli induces DNA double-strand breaks in eukaryotic cells. Science 313:848-51.

9. Chankhamjon P, Javdan B, Lopez J, Hull R, Chatterjee S, Donia MS. 2019. Systematic mapping of drug metabolism by the human gut microbiome. doi:10.1101/538215 \%J bioRxiv:538215.

10. Valm AM, Mark Welch JL, Rieken CW, Hasegawa Y, Sogin ML, Oldenbourg R, Dewhirst FE, Borisy GG. 2011. Systems-level analysis of microbial community organization through combinatorial labeling and spectral imaging. Proc Natl Acad Sci U S A 108:41527.

11. Stacy A, Everett J, Jorth P, Trivedi U, Rumbaugh KP, Whiteley M. 2014. Bacterial fightand-flight responses enhance virulence in a polymicrobial infection. Proc Natl Acad Sci U S A 111:7819-24.

12. Mark Welch JL, Rossetti BJ, Rieken CW, Dewhirst FE, Borisy GG. 2016. Biogeography of a human oral microbiome at the micron scale. Proc Natl Acad Sci U S A 113:E791-800.

13. Jorth $P$, Staudinger BJ, Wu X, Hisert KB, Hayden H, Garudathri J, Harding CL, Radey MC, Rezayat A, Bautista G, Berrington WR, Goddard AF, Zheng C, Angermeyer A, Brittnacher MJ, Kitzman J, Shendure J, Fligner CL, Mittler J, Aitken ML, Manoil C, Bruce JE, Yahr TL, Singh PK. 2015. Regional Isolation Drives Bacterial Diversification within Cystic Fibrosis Lungs. Cell Host Microbe 18:307-19. 
14. Dickson RP, Erb-Downward JR, Freeman CM, McCloskey L, Beck JM, Huffnagle GB, Curtis JL. 2015. Spatial Variation in the Healthy Human Lung Microbiome and the Adapted Island Model of Lung Biogeography. Ann Am Thorac Soc 12:821-30.

15. Chung H, Lieberman TD, Vargas SO, Flett KB, McAdam AJ, Priebe GP, Kishony R. 2017. Global and local selection acting on the pathogen Stenotrophomonas maltophilia in the human lung. Nat Commun 8:14078.

16. Garg N, Wang M, Hyde E, da Silva RR, Melnik AV, Protsyuk I, Bouslimani A, Lim YW, Wong R, Humphrey G, Ackermann G, Spivey T, Brouha SS, Bandeira N, Lin GY, Rohwer F, Conrad DJ, Alexandrov T, Knight R, Dorrestein PC. 2017. Three-Dimensional Microbiome and Metabolome Cartography of a Diseased Human Lung. Cell host \& microbe 22:705716 e4.

17. Aurora P, Wade A, Whitmore P, Whitehead B. 2000. A model for predicting life expectancy of children with cystic fibrosis. Eur Respir J 16:1056-60.

18. Szwed A, John A, Gozdzik-Spychalska J, Czainski W, Czerniak W, Ratajczak J, BaturaGabryel H. 2018. Survival of Patients with Cystic Fibrosis Depending on Mutation Type and Nutritional Status. Adv Exp Med Biol 1023:65-72.

19. Caverly L, Zhao J, LiPuma JJ. 2015. Cystic fibrosis lung microbiome: opportunities to reconsider management of airway infection. Pediatr Pulmonol 50 Suppl 40:S31-8.

20. Bumann D. 2015. Heterogeneous host-pathogen encounters: act locally, think globally. Cell Host Microbe 17:13-9.

21. Zlosnik JE, Zhou G, Brant R, Henry DA, Hird TJ, Mahenthiralingam E, Chilvers MA, Wilcox $P$, Speert DP. 2015. Burkholderia species infections in patients with cystic fibrosis in British Columbia, Canada. 30 years' experience. Ann Am Thorac Soc 12:70-8.

22. Whiteson KL, Bailey B, Bergkessel M, Conrad D, Delhaes L, Felts B, Harris JK, Hunter R, Lim YW, Maughan H, Quinn R, Salamon P, Sullivan J, Wagner BD, Rainey PB. 2014. The upper respiratory tract as a microbial source for pulmonary infections in cystic fibrosis. Parallels from island biogeography. Am J Respir Crit Care Med 189:1309-15.

23. Rogers GB, Marsh P, Stressmann AF, Allen CE, Daniels TV, Carroll MP, Bruce KD. 2010. The exclusion of dead bacterial cells is essential for accurate molecular analysis of clinical samples. Clin Microbiol Infect 16:1656-8.

24. Willner D, Haynes MR, Furlan M, Schmieder R, Lim YW, Rainey PB, Rohwer F, Conrad D. 2012. Spatial distribution of microbial communities in the cystic fibrosis lung. ISME J 6:471-4.

25. Goddard AF, Staudinger BJ, Dowd SE, Joshi-Datar A, Wolcott RD, Aitken ML, Fligner CL, Singh PK. 2012. Direct sampling of cystic fibrosis lungs indicates that DNA-based analyses of upper-airway specimens can misrepresent lung microbiota. Proc Natl Acad Sci U S A 109:13769-74.

26. Coburn B, Wang PW, Diaz Caballero J, Clark ST, Brahma V, Donaldson S, Zhang Y, Surendra A, Gong Y, Elizabeth Tullis D, Yau YC, Waters VJ, Hwang DM, Guttman DS. 2015. Lung microbiota across age and disease stage in cystic fibrosis. Sci Rep 5:10241.

27. Quinn RA, Whiteson K, Lim YW, Zhao J, Conrad D, LiPuma JJ, Rohwer F, Widder S. 2016. Ecological networking of cystic fibrosis lung infections. NPJ Biofilms Microbiomes 2:4.

28. Vazquez-Baeza Y, Pirrung M, Gonzalez A, Knight R. 2013. EMPeror: a tool for visualizing high-throughput microbial community data. Gigascience 2:16. 
29. Hassett DJ, Cuppoletti J, Trapnell B, Lymar SV, Rowe JJ, Yoon SS, Hilliard GM, Parvatiyar K, Kamani MC, Wozniak DJ, Hwang SH, McDermott TR, Ochsner UA. 2002. Anaerobic metabolism and quorum sensing by Pseudomonas aeruginosa biofilms in chronically infected cystic fibrosis airways: rethinking antibiotic treatment strategies and drug targets. Adv Drug Deliv Rev 54:1425-43.

30. Wang M, Carver JJ, Phelan VV, Sanchez LM, Garg N, Peng Y, Nguyen DD, Watrous J, Kapono CA, Luzzatto-Knaan T, Porto C, Bouslimani A, Melnik AV, Meehan MJ, Liu WT, Crusemann M, Boudreau PD, Esquenazi E, Sandoval-Calderon M, Kersten RD, Pace LA, Quinn RA, Duncan KR, Hsu CC, Floros DJ, Gavilan RG, Kleigrewe K, Northen T, Dutton RJ, Parrot D, Carlson EE, Aigle B, Michelsen CF, Jelsbak L, Sohlenkamp C, Pevzner P, Edlund A, McLean J, Piel J, Murphy BT, Gerwick L, Liaw CC, Yang YL, Humpf HU, Maansson M, Keyzers RA, Sims AC, Johnson AR, Sidebottom AM, Sedio BE, et al. 2016. Sharing and community curation of mass spectrometry data with Global Natural Products Social Molecular Networking. Nat Biotechnol 34:828-837.

31. Watrous J, Roach P, Alexandrov T, Heath BS, Yang JY, Kersten RD, van der Voort M, Pogliano K, Gross H, Raaijmakers JM, Moore BS, Laskin J, Bandeira N, Dorrestein PC. 2012. Mass spectral molecular networking of living microbial colonies. Proc Natl Acad Sci U S A 109:E1743-52.

32. Quinn RA, Phelan VV, Whiteson KL, Garg N, Bailey BA, Lim YW, Conrad DJ, Dorrestein PC, Rohwer FL. 2016. Microbial, host and xenobiotic diversity in the cystic fibrosis sputum metabolome. ISME J 10:1483-98.

33. Whitaker P, Meng X, Lavergne SN, El-Ghaiesh S, Monshi M, Earnshaw C, Peckham D, Gooi J, Conway S, Pirmohamed M, Jenkins RE, Naisbitt DJ, Park BK. 2011. Mass spectrometric characterization of circulating and functional antigens derived from piperacillin in patients with cystic fibrosis. J Immunol 187:200-11.

34. Amir A, McDonald D, Navas-Molina JA, Kopylova E, Morton JT, Zech Xu Z, Kightley EP, Thompson LR, Hyde ER, Gonzalez A, Knight R. 2017. Deblur Rapidly Resolves SingleNucleotide Community Sequence Patterns. mSystems 2:e00191-16.

35. Quinn RA, Lim YW, Mak TD, Whiteson K, Furlan M, Conrad D, Rohwer F, Dorrestein P. 2016. Metabolomics of pulmonary exacerbations reveals the personalized nature of cystic fibrosis disease. PeerJ 4:e2174.

36. Lepine F, Milot S, Deziel E, He J, Rahme LG. 2004. Electrospray/mass spectrometric identification and analysis of 4-hydroxy-2-alkylquinolines (HAQs) produced by Pseudomonas aeruginosa. J Am Soc Mass Spectrom 15:862-9.

37. Frydenlund Michelsen $C$, Hossein Khademi SM, Krogh Johansen $H$, Ingmer $H$, Dorrestein PC, Jelsbak L. 2016. Evolution of metabolic divergence in Pseudomonas aeruginosa during long-term infection facilitates a proto-cooperative interspecies interaction. ISME J 10:1323-36.

38. Kakinuma R, Moriyama N, Muramatsu Y, Gomi S, Suzuki M, Nagasawa H, Kusumoto M, Aso T, Muramatsu Y, Tsuchida T, Tsuta K, Maeshima AM, Tochigi N, Watanabe S, Sugihara N, Tsukagoshi S, Saito Y, Kazama M, Ashizawa K, Awai K, Honda O, Ishikawa H, Koizumi N, Komoto D, Moriya H, Oda S, Oshiro Y, Yanagawa M, Tomiyama N, Asamura H. 2015. Ultra-High-Resolution Computed Tomography of the Lung: Image Quality of a Prototype Scanner. PLoS One 10:e0137165. 
39. Hu W, Lum GZ, Mastrangeli M, Sitti M. 2018. Small-scale soft-bodied robot with multimodal locomotion. Nature 554:81-85.

40. Lozupone CA, Hamady M, Kelley ST, Knight R. 2007. Quantitative and qualitative beta diversity measures lead to different insights into factors that structure microbial communities. Appl Environ Microbiol 73:1576-85.

41. Pluskal T, Castillo S, Villar-Briones A, Oresic M. 2010. MZmine 2: modular framework for processing, visualizing, and analyzing mass spectrometry-based molecular profile data. BMC Bioinformatics 11:395.

42. Dieterle F, Ross A, Schlotterbeck G, Senn H. 2006. Probabilistic quotient normalization as robust method to account for dilution of complex biological mixtures. Application in $1 \mathrm{H}$ NMR metabonomics. Anal Chem 78:4281-90.

43. Gonzalez A, Navas-Molina JA, Kosciolek T, McDonald D, Vazquez-Baeza Y, Ackermann G, DeReus J, Janssen S, Swafford AD, Orchanian SB, Sanders JG, Shorenstein J, Holste H, Petrus S, Robbins-Pianka A, Brislawn CJ, Wang M, Rideout JR, Bolyen E, Dillon M, Caporaso JG, Dorrestein PC, Knight R. 2018. Qiita: rapid, web-enabled microbiome meta-analysis. Nat Methods 15:796-798.

44. Edgar RC. 2010. Search and clustering orders of magnitude faster than BLAST. Bioinformatics 26:2460-1.

45. Eisenhofer R, Minich JJ, Marotz C, Cooper A, Knight R, Weyrich LS. 2019. Contamination in Low Microbial Biomass Microbiome Studies: Issues and Recommendations. Trends Microbiol 27:105-117.

46. Minich JJ, Sanders JG, Amir A, Humphrey G, Gilbert J, Knight R. 2019. Quantifying and understanding well-to-well contamination in microbiome research. doi:10.1101/577718 \%J bioRxiv:577718.

47. Caporaso JG, Kuczynski J, Stombaugh J, Bittinger K, Bushman FD, Costello EK, Fierer N, Pena AG, Goodrich JK, Gordon JI, Huttley GA, Kelley ST, Knights D, Koenig JE, Ley RE, Lozupone CA, McDonald D, Muegge BD, Pirrung M, Reeder J, Sevinsky JR, Turnbaugh PJ, Walters WA, Widmann J, Yatsunenko T, Zaneveld J, Knight R. 2010. QIIME allows analysis of high-throughput community sequencing data. Nat Methods 7:335-6.

48. Lozupone C, Knight R. 2005. UniFrac: a new phylogenetic method for comparing microbial communities. Appl Environ Microbiol 71:8228-35.

49. Protsyuk I, Melnik AV, Nothias LF, Rappez L, Phapale P, Aksenov AA, Bouslimani A, Ryazanov S, Dorrestein PC, Alexandrov T. 2018. 3D molecular cartography using LC-MS facilitated by Optimus and 'ili software. Nat Protoc 13:134-154. 